\title{
Asymptotic behavior of tidal damping in alluvial estuaries
}

\author{
Huayang $\mathrm{Cai}^{1}$ and Hubert H. G. Savenije ${ }^{1}$ \\ Received 31 January 2013; revised 14 October 2013; accepted 20 October 2013; published 15 November 2013.
}

[1] Tidal wave propagation can be described analytically by a set of four implicit equations, i.e., the phase lag equation, the scaling equation, the damping equation, and the celerity equation. It is demonstrated that this system of equations has an asymptotic solution for an infinite channel, reflecting the balance between friction and channel convergence.

Subsequently, explicit expressions for the tidal amplitude and velocity amplitude are derived, which are different from the generally assumed exponential damping equation that follows from linearizing the friction term. Analysis of the asymptotic behavior demonstrates that exponential damping of the tidal amplitude is only correct for a frictionless wave or an ideal estuary (no damping). However, in estuaries with modest damping (near ideal) it provides a reasonable approximation. In natural estuaries, there is generally a need to take account of local variability of, e.g., depth and friction, by subdividing the estuary into multiple reaches. This is illustrated with an example of the Scheldt estuary, which has been gradually deepened for navigation purpose over the last half century. The analytical model is used to study the effect of this deepening on the tidal dynamics in the main navigation channel, demonstrating that the navigation channel will become "overamplified" when it reaches a depth larger than the critical depth. In the case of overamplification, a further increase of the depth reduces the amplification until critical convergence (condition for a frictionless standing wave) is reached asymptotically. Finally, based on the ratio between the tidal amplitude at the seaward boundary and the asymptotic tidal amplitude, estuaries can be classified into damped, amplified, or ideal estuaries, which is illustrated with 23 real estuaries.

Citation: Cai, H., and H. H. G. Savenije (2013), Asymptotic behavior of tidal damping in alluvial estuaries, J. Geophys. Res. Oceans, 118, 6107-6122, doi:10.1002/2013JC008772.

\section{Introduction}

[2] Knowledge of tidal dynamics is essential to analyze the effect of human interventions, such as dredging for navigation, fresh water withdrawal, and design of regulation structures, in estuaries. Analytical tools are invaluable tools to assess the impact of such interventions as they provide direct insight in cause-effect relations that are generally nonlinear. Over time, a range of 1-D analytical solutions of the tidal dynamics equations have been derived by: Hunt [1964], Dronkers [1964], Ippen [1966], Jay [1991], Friedrichs and Aubrey [1994], Lanzoni and Seminara [1998], Savenije [1992a, 1998, 2001, 2005, 2012], Prandle [2003], Savenije and Veling [2005], Souza and Hill [2006], Savenije et al. [2008], Friedrichs [2010], Toffolon and Savenije Additional supporting information may be found in the online version of
this article.
${ }^{1}$ Department of Water Management, Faculty of Civil Engineering and
Geosciences, Delft University of Technology, Delft, Netherlands

Corresponding author: H. H. G. Savenije, Department of Water Management, Faculty of Civil Engineering and Geosciences, Delft University of Technology, Stevinweg 1, PO Box 5048, NL-2600 GA Delft, Netherlands. (h.h.g.savenije@tudelft.nl)

(C2013. American Geophysical Union. All Rights Reserved. 2169-9275/13/10.1002/2013JC008772
[2011], Van Rijn [2011], and Cai et al. [2012a]. All these solutions invariably require certain assumptions on estuary shape and flow characteristics. In this study, we focus on analytical solutions for infinite length estuaries (long coastal plain estuaries), where we may assume that the ratio of tidal amplitude to velocity amplitude is constant. Moreover, since the 1-D partial differential equations (the St. Venant equations) are nonlinear, analytical solutions require some form of linearization. Most researchers linearized the equations by means of perturbation analysis in an Eulerian framework and linearized the friction term. In contrast, Savenije [1998] used a Lagrangian approach and derived a nonlinear damping equation by subtracting high water (HW) and low water (LW) envelopes that retained both the quadratic velocity in the numerator and the periodic variation of the hydraulic radius in the denominator. This method, termed the envelope method, is a quasinonlinear approach because it makes use of a linear harmonic function to represent the Lagrangian flow velocity, which is an implicit way of linearization. On the basis of this quasi-nonlinear approach, Savenije et al. [2008] provided a fully explicit solution of the tidal hydraulic equations, by solving the set of four implicit equations for the velocity scale, the damping, the phase lag, and the wave celerity.

[3] Recently, Cai et al. [2012a] proposed a general analytical framework to simulate tidal wave propagation, 

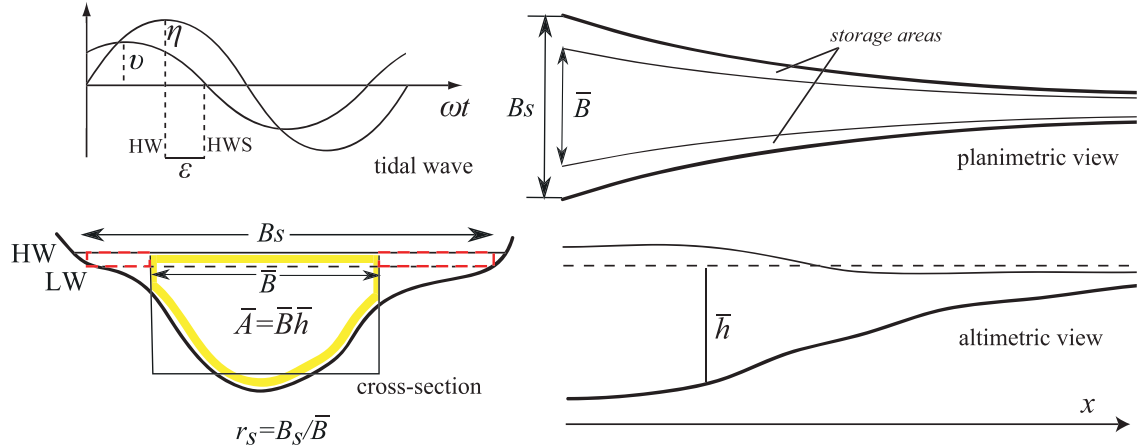

Figure 1. Sketch of the geometry of the idealized tidal channel and notation: tidal oscillations of water level and velocity and definition of the phase lag $\varepsilon$; definition of the equivalent rectangular cross section of width $B$, and of the total width $B_{S}$ including storage areas; planimetric view of the estuary with storage areas; lateral view showing instantaneous and tidally averaged depth.

which allowed the comparison of different analytical approaches, mentioned above and concluded that the main difference between these models lies in the treatment of the friction term. It appeared that linear solutions, such as those by Toffolon and Savenije [2011] or Van Rijn [2011], based on the classical Lorentz linearization [Lorentz, 1926] for the friction term, are identical, although they used a different solution method. It is shown by Cai et al. [2012a] that a hybrid combination of the traditional linearized approach [e.g., Toffolon and Savenije, 2011] and the envelope method [e.g., Savenije et al., 2008] provides the most accurate predictive results. However, although they provided an explicit solution for tidal damping, they were not yet able to write the tidal amplitude and velocity amplitude as explicit functions of distance. In this paper, we provide a new explicit solution for the tidal amplitude. Furthermore, we demonstrate that, in contrast with what is generally believed, the tidal amplitude has an asymptotic solution. Classical methods, assuming exponential damping, either lead to an infinite amplitude (when amplified), or a zero amplitude (when damped), but here we show that the asymptotic solution corresponds with the amplitude of an ideal estuary.

[4] The paper is organized as follows. First, a comparison between classical solutions and those developed by Savenije et al. [2008], Toffolon and Savenije [2011], and Cai et al. [2012a] is presented in section 2. In section 3, a fully explicit solution of the tidal damping equation is developed, leading to an explicit equation for the tidal amplitude. In section 4, the upstream and downstream asymptotic behavior is explored based on the obtained explicit solution. In section 5, the model is compared to observations in the Scheldt estuary, which over the last half century has been substantially deepening with drastic implications for the tidal dynamics. The asymptotic solution is subsequently used to classify 23 real estuaries in the world. Finally, conclusions are drawn in section 6.

\section{Comparison of Models}

[5] A conceptual sketch of the geometry of an idealized tidal channel is presented in Figure 1, together with a simplified picture of the periodic oscillations of water level and velocity defining the phase lag. We consider a tidal channel with varying width and depth with a large width to depth ratio, so that it may be approximated by a rectangular cross section, and with lateral storage areas, described by the storage width ratio $r_{S}=B_{S} / \bar{B}$, i.e., the ratio between the storage width $B_{S}$ and the stream width $\bar{B}$ (see Figure 1).

[6] In alluvial estuaries, the tidally averaged crosssectional area $\bar{A}=\bar{B} h$ can be described by an exponential function [e.g., Savenije, 2005, 2012]:

$$
\bar{A}=\overline{A_{0}} \exp (-x / a)
$$

where $x$ is the longitudinal coordinate directed landward, $\overline{A_{0}}$ is the cross-sectional area at the estuary mouth, $\bar{h}$ is the tidal average depth of flow, and $a$ is the convergence length of the cross-sectional area.

[7] The system is forced by a sinusoidal tidal wave with a tidal period $T$ and a frequency $\omega=2 \pi / T$. As the wave propagates into the estuary, it has a wave celerity $c$, an amplitude of the tidal water level variation $\eta$, a tidal velocity amplitude $v$, and a phase lag $\varepsilon$, defined as the phase difference between high water (HW) and high water slack (HWS) or between low water (LW) and low water slack (LWS). For a simple harmonic wave, $\varepsilon=\pi / 2-\left(\phi_{z}-\phi_{U}\right)$, where $\phi_{z}$ is the phase of water level and $\phi_{U}$ the phase of the tidal velocity.

[8] Table 1 presents one consistent theoretical framework for the solution of the one-dimensional hydrodynamic equations for tidal wave propagation as provided by $\mathrm{Cai}$ et al. [2012a] (based on Toffolon et al. [2006] and Savenije et al. [2008]). Cai et al. [2012a] showed that different friction formulations can be used in the envelope method to arrive at an equal number of analytical solutions. In general, the main classes of the solutions are: (1) quasinonlinear solution with nonlinear friction term [Savenije et al., 2008], (2) modified linear solution with Lorentz's linearization [Lorentz, 1926], (3) hybrid solution characterized by a weighted average of Lorentz's linearization, with weight $1 / 3$, and the nonlinear friction term, with weight $2 / 3$ [Cai et al., 2012a]. And the solutions can be obtained by solving four implicit equations, i.e., the phase lag equation (T1), scaling equation (T2), celerity equation (T3), and damping equation (T4a, T4b, or T4c), where $\delta$ is the damping number (a dimensionless description of the 
Table 1. Analytical Framework for Tidal Wave Propagation [Cai et al., 2012a]

\begin{tabular}{|c|c|c|c|c|c|}
\hline \multicolumn{2}{|c|}{ Case } & Phase lag $\tan (\varepsilon)$ & Scaling $\mu$ & Celerity $\lambda^{2}$ & Damping $\delta$ \\
\hline General & $\begin{array}{l}\text { Quasi-nonlinear } \\
\text { Modified linear } \\
\text { Hybrid }\end{array}$ & $\lambda /(\gamma-\delta)(\mathrm{T} 1)$ & $\cos (\varepsilon) /(\gamma-\delta)(\mathrm{T} 2)$ & $1-\delta(\gamma-\delta)(\mathrm{T} 3)$ & $\begin{array}{l}\gamma / 2-\chi \mu^{2} / 2(\mathrm{~T} 4 \mathrm{a}) \\
\gamma / 2-4 \chi \mu /(3 \pi \lambda)(\mathrm{T} 4 \mathrm{~b}) \\
\gamma / 2-4 \chi \mu^{2} /(9 \pi \lambda)-\chi \mu^{2} / 3(\mathrm{~T} 4 \mathrm{c})\end{array}$ \\
\hline Constant cross section & $\begin{array}{l}\text { Quasi-nonlinear } \\
\text { Modified linear } \\
\text { Hybrid }\end{array}$ & $-\lambda / \delta$ & $-\cos (\varepsilon) / \delta$ & $1+\delta^{2}$ & $\begin{array}{l}-\chi \mu^{2} / 2 \\
-4 \chi \mu /(3 \pi \lambda) \\
-4 \chi \mu^{2} /(9 \pi \lambda)-\chi \mu^{2} / 3\end{array}$ \\
\hline Frictionless $(\gamma<2)$ & & $\sqrt{4 / \gamma^{2}-1}$ & 1 & $1-\gamma^{2} / 4$ & $\gamma / 2$ \\
\hline Frictionless $(\gamma \geq 2)$ & & 0 & $\left(\gamma-\sqrt{\gamma^{2}-4}\right) / 2$ & 0 & $\left(\gamma-\sqrt{\gamma^{2}-4}\right) / 2$ \\
\hline Ideal estuary & & $1 / \gamma$ & $\sqrt{1 /\left(1+\gamma^{2}\right)}$ & 1 & 0 \\
\hline
\end{tabular}

amplification $(\delta>0)$ or damping $(\delta<0)$ of the tidal wave amplitude along the estuary), $\mu$ the velocity number (the actual velocity scaled with the frictionless value in a prismatic channel), $\gamma$ the estuary shape number (representing the effect of cross-sectional convergence), $\chi$ the friction number (describing the role of the frictional dissipation), and $\lambda$ the celerity number (the ratio between the theoretical frictionless celerity in a prismatic channel and the actual wave celerity). The dimensionless variables of these equations are presented in Table 2, where $c_{0}$ is the classical wave celerity of a frictionless wave in a prismatic channel:

$$
c_{0}=\sqrt{g \bar{h} / r_{S}}
$$

and $f$ is the dimensionless friction factor following from the envelope method [Savenije, 1998], defined as:

$$
f=g /\left(K^{2} \bar{h}^{1 / 3}\right)\left[1-(4 \zeta / 3)^{2}\right]^{-1},
$$

where $g$ is the acceleration due to gravity, $K$ is the ManningStrickler friction coefficient. The factor $4 / 3$ stems from a Taylor approximation of the exponent of the hydraulic radius in the friction term. The analytical solutions for some particular cases, including: constant cross section $(\gamma=0)$, frictionless channel $(\chi=0$, both with subcritical convergence $(\gamma<2)$ and supercritical convergence $(\gamma \geq 2))$ and ideal estuary $(\delta=0)$, are also presented in Table $\overline{1}$. For a frictionless or an ideal estuary, all methods are identical.

[9] Savenije et al. [2008] showed that these equations (corresponding with quasi-nonlinear model in Table 1) can be solved explicitly, with two families of solutions. The first family consists of a mixed tidal wave with $0<\varepsilon<\pi / 2$, while the second family consists of an "appa-

Table 2. The Definition of Dimensionless Parameters

\begin{tabular}{ll}
\hline \multicolumn{1}{c}{ Dimensionless Parameters } \\
\hline $\begin{array}{l}\text { Independent } \\
\text { Tidal amplitude at the } \\
\text { downstream boundary, } \\
\zeta_{0}=\eta_{0} / \bar{h}\end{array}$ & Dependent \\
$\begin{array}{ll}\text { Estuary shape, } \gamma=c_{0} /(\omega a) \\
\begin{array}{l}\text { Friction number at the } \\
\text { downstream boundary, }\end{array}\end{array}$ & Delerity number, $\lambda=c_{0} / c$ \\
$\chi_{0}=r_{S} f c_{0} \zeta_{0} /(\omega \bar{h})$ & Phase lag, $\varepsilon=\pi / 2-\left(\phi_{z}-\phi_{U}\right)$ \\
\hline
\end{tabular}

rently standing" wave $(\varepsilon=0)$. Recently, Toffolon and Savenije [2011] modified the classical linearized solution for tidal hydrodynamics in convergent channels by exploiting an iterative procedure to determine friction and a multireach approach (corresponding with modified linear model in Table 1). It was demonstrated by Cai et al. [2012a] that the modified linear model overestimates the tidal damping while the quasi-nonlinear model underestimates it, and the hybrid model provides the best predictions when compared with numerical results. Figure 2 describes the variation of the main dependent dimensionless parameters as a function of shape number $\gamma$ and friction number $\chi$, obtained with different analytical models. Unlike the discontinuous behavior (i.e., with two families of solutions) and the transition toward a standing wave (i.e., the wave celerity approaching infinity) predicted by Savenije et al. [2008], both linear and hybrid models provide a continuous solution in the transition zone of critical convergence [Jay, 1991] where $\gamma$ is close to 2. This is important since it enables the linear and hybrid models to be applicable in the zones where convergence exceeds critical convergence.

[10] It is important to note that the two independent variables $\gamma$ and $\chi$ depend on the tidally averaged depth $\bar{h}$ and tidal amplitude to depth ratio $\zeta$, respectively. In Figure 2, we adopted a multireach approach in which the damping number $\delta$ is integrated in short reaches over which the estuary shape number $\gamma$ and friction number $\chi$ are considered constant. This is done by simple explicit integration of the linear differential equation over a distance $\Delta x($ e.g., $1 \mathrm{~km})$, leading to tidal amplitude at a distance $\Delta x$ upstream, which is repeated for the whole estuary [Savenije et al., 2008]. It should be noted that similar multireach approaches for the representation of topography and friction have been commonly used in literature [e.g., Jay and Flinchem, 1997; Toffolon and Savenije, 2011].

[11] The classical analytical solution to tides in infinite channels assumes the tidal wave to be exponentially damped (or amplified) as it progresses into the estuary [e.g., Hunt, 1964; Ippen, 1966; Friedrichs and Aubrey, 1994; Friedrichs, 2010; Van Rijn, 2011], where the tidal amplitude and velocity amplitude, rewritten in our notation, are given by:

$$
\begin{aligned}
\eta & =\eta_{0} \exp \left(x \delta \omega / c_{0}\right), \\
v & =v_{0} \exp \left(x \delta \omega / c_{0}\right),
\end{aligned}
$$

where $\eta_{0}, v_{0}$ represent the tidal amplitude and velocity amplitude at the estuary mouth, respectively. 

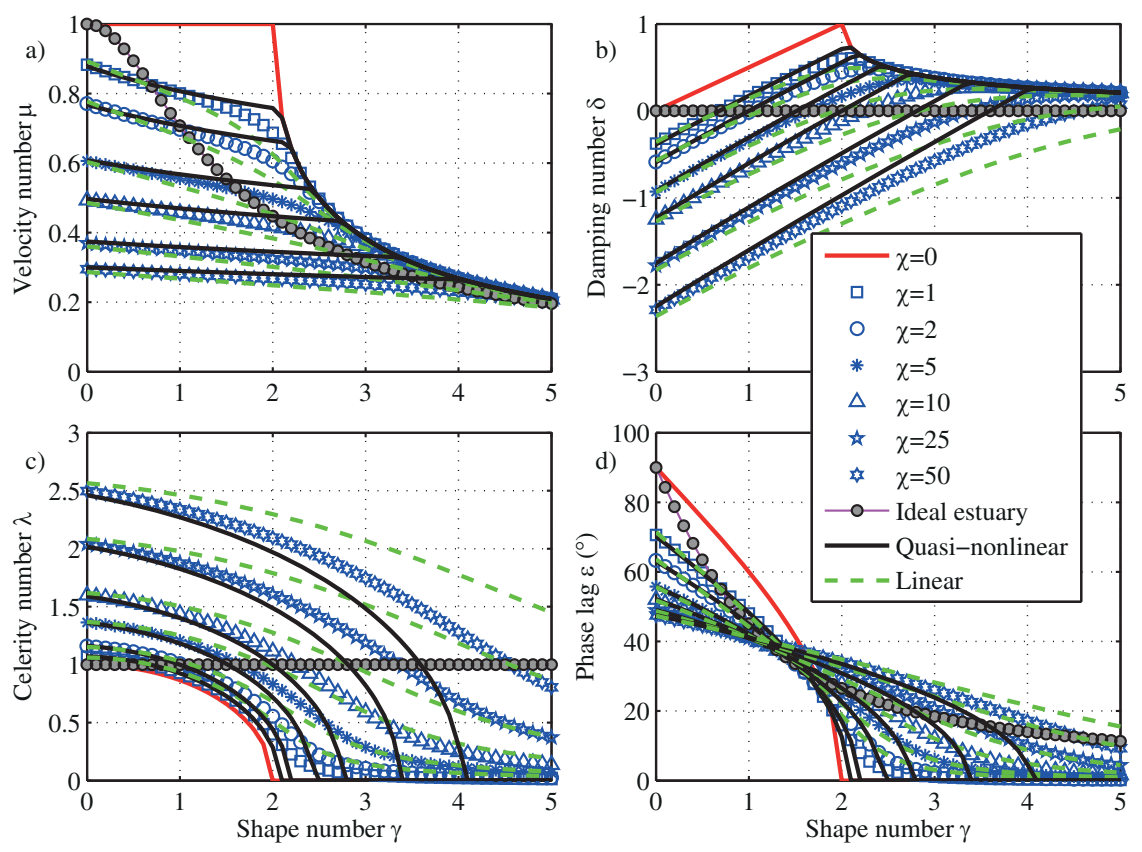

Figure 2. Main dimensionless parameters: (a) the velocity number $\mu$, (b) damping number $\delta$, (c) celerity number $\lambda$, and (d) phase lag $\varepsilon$ obtained with various analytical relationships as a function of estuary shape number $\gamma$ for different values of friction number $\chi$. The gray symbols represent the ideal estuary (see Table 1).

[12] Although widely applied, it can be shown that this assumption is only valid if the friction number $\chi$ is constant along the estuary axis (i.e., $\chi=\chi_{0}$ ), where $\chi_{0}$ is the friction number calculated at the estuary mouth. This can be seen from Figure 3, which shows the dimensionless tidal amplitude $\eta^{*}$ as a function of dimensionless distance $x^{*}$, using the quasi-nonlinear method of Savenije et al. [2008], indicated by (Q), modified linear method of Toffolon and Savenije [2011], indicated by (M), and hybrid method of Cai et al. [2012a], indicated by (H). It can be seen that these solutions only coincide with the classical equation if we use a constant friction number $\chi$ (indicated by $\mathrm{a}, \mathrm{b}$, and $\mathrm{c}$ ), where the definitions of $\eta^{*}$ and $x^{*}$ are:

$$
\eta^{*}=\eta / \eta_{0}, \quad x^{*}=x \omega / c_{0} .
$$

[13] It is worth noting that in the modified linear model the authors also used exponential damping, but that as a result of the iterative multireach approach with variable friction, the error made by assuming exponential damping was small (the linear model being a good first-order approximation).

[14] An important difference between the classical solution and the quasi-nonlinear approach is that the latter results in an equilibrium amplitude as an asymptotic solution when approaching infinity, whereas the classical solution approaches zero for a damped wave and infinity for an amplified wave. This asymptotic solution implies that the flow adapts itself to the shape of the estuary until it has the same properties as an ideal estuary, with a constant friction and tidal amplitude.

[15] The condition of an ideal estuary (no damping) is easily set by imposing $\delta=0$, whereafter the relationship between the friction number $\chi$ and the shape number $\gamma$ in the hybrid model becomes (see T4c in Table 1):

$$
\chi=\gamma /\left\{8 /\left(9 \pi \sqrt{1+\gamma^{2}}\right)+2 /\left[3\left(1+\gamma^{2}\right)\right]\right\} .
$$

[16] In the quasi-nonlinear model, this relationship reads (see T4a in Table 1):

$$
\chi=\gamma\left(\gamma^{2}+1\right)
$$

[17] Similarly, in the linearized model, it reads (see T4b in Table 1):

$$
\chi=3 \pi \gamma \sqrt{\gamma^{2}+1} / 8
$$

[18] It is worth noting that these methods use different definitions of the dimensionless friction factor $f$ (i.e., equation 3) incorporated in the friction number $\chi$. The Lorentz's linearization considers a time-invariant depth in the friction term, which is the same as taking $\zeta=0$ in (3), i.e.f $f \approx g /\left(K^{2} \bar{h}^{1 / 3}\right)$ [Toffolon et al., 2006; Toffolon and Savenije, 2011].

[19] Using the definition of the friction number $\chi$ (see Table 2) in equation 7 yields the expression of the asymptotic tidal amplitude for the hybrid model [Cai et al., 2012a]:

$$
\begin{gathered}
\eta_{\mathrm{inf}}=\frac{-9 m_{1}+3 \sqrt{9 m_{1}^{2}+64 m_{2}^{2}}}{32 m_{2}} \bar{h}, \\
m_{1}=r_{s} g c_{0},
\end{gathered}
$$




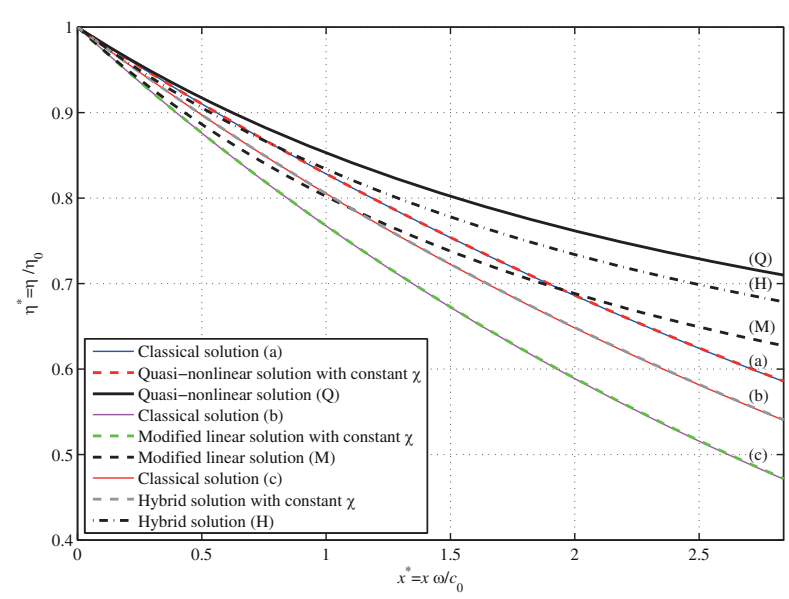

Figure 3. Longitudinal variation of tidal amplitude obtained with quasi-nonlinear (Q), modified linear (M), and hybrid $(\mathrm{H})$ models applying constant and variable friction number for $\gamma=1, \zeta_{0}=0.1, K=30 \mathrm{~m}^{1 / 3} \mathrm{~s}^{-1}$. For comparison, the three classical solutions have been calculated with the boundary conditions for $\delta_{0}$ corresponding to (a) the quasinonlinear model, (b) the modified linear model, and (c) the hybrid model.

$$
m_{2}=\gamma K^{2} \bar{h}^{4 / 3} \omega /\left\{8 /\left(9 \pi \sqrt{1+\gamma^{2}}\right)+2 /\left[3\left(1+\gamma^{2}\right)\right]\right\} .
$$

[20] For small tidal amplitude to depth ratio $(\zeta \ll 1)$, so that $f \approx g /\left(K^{2} \bar{h}^{1 / 3}\right)$, equation 7 can be simplified whereby $\chi$ is linear in $\zeta$. As a result, equation 10 modifies into:

$$
\eta_{\text {inf }} \approx m_{2} \bar{h} / m_{1} \text {. }
$$

[21] The relationship found for the quasi-nonlinear model is similar to equation 13 with only a different expression for $m_{2}$ :

$$
m_{2}=\gamma\left(\gamma^{2}+1\right) K^{2} \bar{h}^{4 / 3} \omega
$$

[22] Similarly, the expression of $m_{2}$ for the linear model reads:

$$
m_{2}=3 \pi \gamma \sqrt{\gamma^{2}+1} K^{2} \bar{h}^{4 / 3} \omega / 8 .
$$

[23] An example of the asymptotic solutions of these models is given in Figure 4 (the MATLAB scripts are provided as supporting information). We can see that the three solutions only differ in the parameter $m_{2}$ (i.e., $8 \sqrt{1+\gamma^{2}} /(9 \pi)+2 / 3$ for the quasi-nonlinear model and $\pi /\left(4 \sqrt{1+\gamma^{2}}\right)+1 / 3$ for the modified linear model $)$, resulting in a slightly different asymptotic value.

\section{Explicit Solution to the Tidal Damping Equation}

[24] In principle, the explicit solution to the tidal damping equation can be derived for all three analytical models, i.e., quasi-nonlinear, modified linear, and hybrid models. In the following, we focus on the hybrid model since it provides the best predictive results [Cai et al., 2012a]. The derivation for the other models are summarized in Appendixes $\mathrm{A}$ and $\mathrm{B}$.

[25] For an infinite length estuary and assuming that the freshwater discharge is small compared to tidal discharge, Cai et al. [2012a] derived an expression for tidal damping or amplification through the envelope method:

$$
\frac{1}{\eta} \frac{\mathrm{d} \eta}{\mathrm{d} x}\left[1+\frac{g \eta}{c v \sin (\varepsilon)}\right]=\frac{1}{a}-\frac{2}{3} f \frac{v}{\bar{h} c}\left[\frac{4}{3 \pi}+\sin (\varepsilon)\right]
$$

which is identical to the dimensionless damping equation (T4c) for $\delta$ in Table 1.

[26] Until now, the tidal amplitude and velocity amplitude variation along the estuary axis were obtained by stepwise numerical integration of the damping number $\delta$. Here, we revisit the analytical approach proposed by Cai et al. [2012a] and derive an explicit analytical solution of the tidal damping equation, requiring the following assumptions: 1 . A constant friction factor: $f 2 ; 2$. a constant phase lag: $\varepsilon ; 3$. a constant wave celerity: $c$; 4. a constant depth: $\bar{h}$; and 5 . the velocity amplitude and tidal amplitude are proportional: $\frac{1}{v} \frac{\partial v}{\partial x}=\frac{1}{\eta} \frac{\partial \eta}{\partial x}$.

[27] The last assumption implies that the ratio of the velocity amplitude to the tidal amplitude is constant, which applies to estuaries of infinite length [Savenije et al., 2008]:

$$
\frac{v}{\eta}=\frac{v_{0}}{\eta_{0}}
$$

[28] This relationship is valid for long coastal plain estuaries, which was demonstrated to be correct by

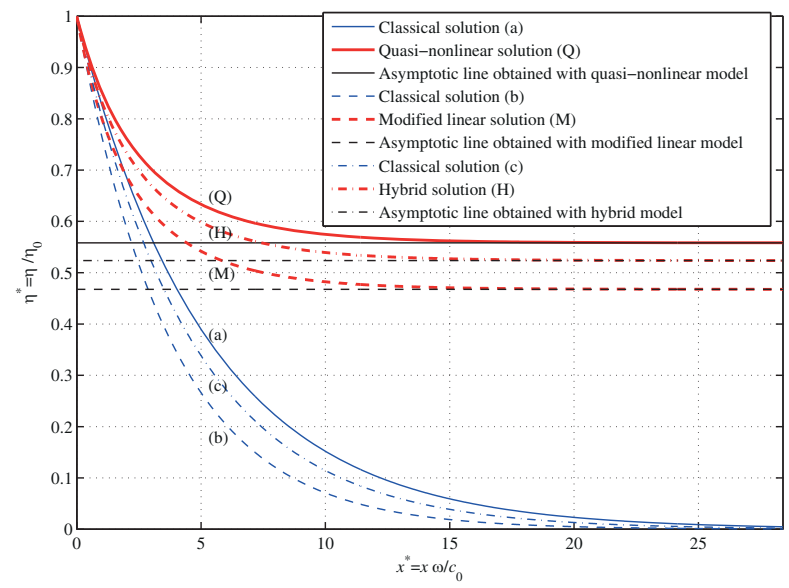

Figure 4. Longitudinal variation of tidal amplitude obtained with quasi-nonlinear $(\mathrm{Q})$, modified linear $(\mathrm{M})$, and hybrid $(\mathrm{H})$ models for $\gamma=1, \zeta_{0}=0.1, K=30 \mathrm{~m}^{1 / 3} \mathrm{~s}^{-1}$. The black lines represent the corresponding asymptotic lines obtained with equation 10 . The blue lines represent the classical solutions for the three different boundary conditions of $\delta_{0}$ corresponding with (a) the quasi-nonlinear model, (b) the modified linear model, and (c) the hybrid model. 
Savenije [1992a, 1993] based on numerical simulations in a wide range of convergent estuaries. Moreover, as a result of the multireach approach, e.g., to account for variability in the geometry, a potential error resulting from this assumption is small.

[29] To simplify equation 16, two parameters are introduced:

$$
\begin{gathered}
\psi=1+\frac{g \eta}{c v \sin \varepsilon}=1+\frac{g \eta_{0}}{c v_{0} \sin \varepsilon}=1+\frac{1}{\mu_{0}^{2}}, \\
\beta=\frac{2}{3} f \frac{v_{0}}{\bar{h} c}\left[\frac{4}{3 \pi}+\sin (\varepsilon)\right] .
\end{gathered}
$$

which are constant under the above assumptions. It can be shown that $\psi \geq 2$ since $0<\mu_{0} \leq 1$ [Savenije et al., 2008], while $\beta>0$.

[30] Substitution of equations (17-19) into equation 16 leads to:

$$
\frac{\mathrm{d} \eta^{*}}{\mathrm{~d} x}=\frac{\eta^{*}}{\psi a}\left(1-a \beta \eta^{*}\right)
$$

[31] It can be seen from equation 20 that convergence and friction are in balance if $\eta^{*}=1 /(a \beta)$, which is the case of an ideal estuary where there is no tidal damping or amplification. In fact, there are two situations where there is no damping. The first one is the trivial situation where $\eta^{*}=0$, and the other is where $\eta^{*}=1 /(a \beta)$.

[32] With $\eta_{0}^{*}=1$ at $x=0$, integration yields an explicit solution for the tidal amplitude:

$$
\eta^{*}=\frac{1}{a \beta+(1-a \beta) \exp [-x /(\psi a)]}=\frac{\eta_{i n f}^{*}}{1-\left(1-\eta_{i n f}^{*}\right) \exp [-x /(\psi a)]},
$$

where the infinite tidal amplitude $\eta_{\text {inf }}^{*}=1 /(a \beta)$ and the damping scale $\psi a$ are constants.

[33] Introducing the dimensionless parameters used in Table 2, equation 21 can be rewritten as:

$$
\eta^{*}=\frac{\gamma /\left[8 \chi_{0} \mu_{0} \lambda /(9 \pi)+2 \chi_{0} \mu_{0}^{2} \lambda^{2} / 3\right]}{1-\left\{1-\gamma /\left[8 \chi_{0} \mu_{0} \lambda /(9 \pi)+2 \chi_{0} \mu_{0}^{2} \lambda^{2} / 3\right]\right\} \exp \left[-\gamma \mu_{0}^{2} x^{*} /\left(1+\mu_{0}^{2}\right)\right]} .
$$

[34] Subsequently, the solutions of tidal amplitude $\eta$ and velocity amplitude $v$ are:

$$
\begin{gathered}
\eta=\eta_{0} \eta^{*} \\
v=\frac{r_{S} c_{0} \mu_{0}}{\bar{h}} \eta=\frac{r_{S} c_{0} \mu_{0}}{\bar{h}} \eta_{0} \eta^{*}
\end{gathered}
$$

where equation 24 has been obtained from the definition of the velocity number in Table 2 .

[35] Figure 5 presents the computed tidal amplitude along the estuary for the case of modest convergence $(\gamma=0.5)$ resulting in a damped tidal wave, and for the case of strong convergence $(\gamma=2)$, resulting in an amplified wave. The drawn lines correspond with the new explicit equation 22 whereas the dashed lines correspond with the classical exponential equation 4 . It can be seen clearly that

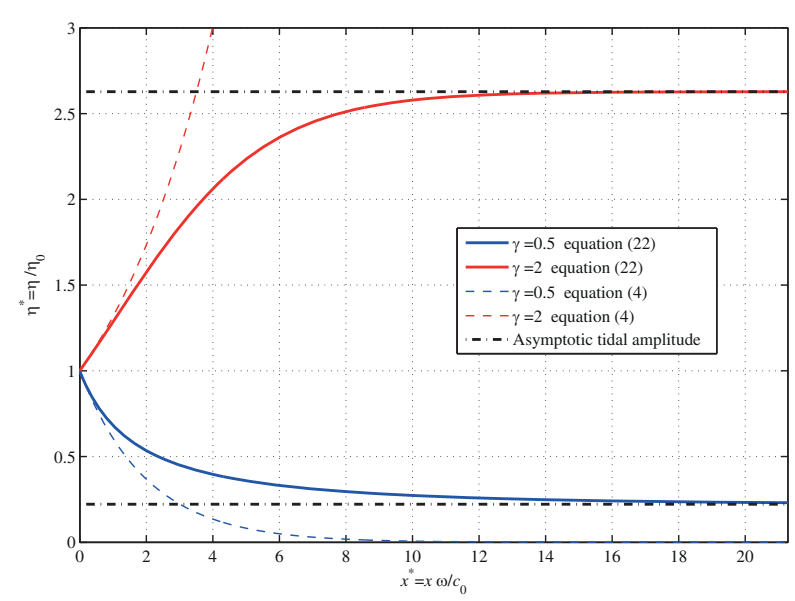

Figure 5. Comparison of longitudinal tidal amplitude between the proposed explicit equation 22 and the classical equation 4 , for strong $(\gamma=2)$ and modest $(\gamma=0.5)$ convergence with $\zeta_{0}=0.1, K=30 \mathrm{~m}^{1 / 3} \mathrm{~s}^{-1}$.

the two approaches have the same asymptote at $x^{*}=0$, but that the difference lies in the asymptote when $x^{*}$ approaches infinity. With equation 4 the tidal amplitude approaches zero for a damped wave and infinity for an amplified wave, whereas equation 22 has an asymptotic tidal amplitude that reflects the balance between friction and channel convergence. However, for an estuary with constant cross-section (i.e., $\gamma=0$ without channel convergence) equation 22 is no longer applicable, but continuous damping leads to an asymptote of $\eta_{\text {inf }}^{*}=0$.

\section{Asymptotic Behavior of the Damping Equation}

\subsection{Upstream Asymptotic Behavior}

[36] In the asymptotic situation with no river discharge, the infinite dimensionless tidal amplitude $\eta_{\text {inf }}^{*}$ reads:

$$
\begin{aligned}
& \eta_{\text {inf }}^{*}=\frac{1}{a \beta}=\gamma /\left[8 \chi_{0} \mu_{0} \lambda /(9 \pi)+2 \chi_{0} \mu_{0}^{2} \lambda^{2} / 3\right] \\
& =\gamma /\left[8 \chi_{0} \mu_{I} \lambda_{I} /(9 \pi)+2 \chi_{0} \mu_{I}^{2} \lambda_{I}^{2} / 3\right]=\frac{\chi_{I}}{\chi_{0}},
\end{aligned}
$$

where the last step in (25) follows from using the expressions for $\chi$ in equation 7 and for $\mu_{0}$ and $\lambda$ of an ideal estuary [Cai et al., 2012a]:

$$
\mu_{I}^{2}=\frac{1}{1+\gamma^{2}}, \quad \lambda_{I}=1,
$$

where the subscript $I$ stands for the ideal estuary. This implies that in the upstream asymptotic situation the amplitude tends to an ideal estuary with constant amplitude. If $\eta_{\text {inf }}^{*}>1$ ( or $\left.\chi_{I}>\chi_{0}, a \beta<1\right)$, then the estuary is amplified; if $\eta_{\text {inf }}^{*}<1$ (or $\chi_{I}<\chi_{0}, a \beta>1$ ), then it is damped; and if $\eta_{\text {inf }}^{*}=1\left(\right.$ or $\left.\chi_{I}=\chi_{0}, a \beta=1\right)$, the estuary is ideal.

[37] Using (7) for $\chi$ of an ideal estuary, the amplitude then becomes: 


$$
\begin{aligned}
\eta_{\text {inf }} & =\frac{\chi_{I}}{\chi_{0}} \eta_{0}=\frac{\gamma /\left\{8 /\left(9 \pi \sqrt{1+\gamma^{2}}\right)+2 /\left[3\left(1+\gamma^{2}\right)\right]\right\}}{\chi_{0}} \eta_{0} \\
& =\frac{\omega}{c_{0}} \frac{\bar{h}^{2}}{r_{S} f} \gamma /\left\{8 /\left(9 \pi \sqrt{1+\gamma^{2}}\right)+2 /\left[3\left(1+\gamma^{2}\right)\right]\right\},
\end{aligned}
$$

or

$$
\zeta_{\text {inf }}=\frac{\bar{h}}{a} \frac{1}{r_{s} f\left\{8 /\left(9 \pi \sqrt{1+\gamma^{2}}\right)+2 /\left[3\left(1+\gamma^{2}\right)\right]\right\}},
$$

which is an expression that only depends on the geometry and the friction, and is independent on the boundary conditions. The caveat is that the equation applies to long (infinite) estuaries where we may assume that $\frac{1}{v} \frac{\partial v}{\partial x}=\frac{1}{\eta} \frac{\partial \eta}{\partial x}$. We can see that in deep convergent estuaries (with large $\gamma$ ), this can lead to a large equilibrium amplitude. Equation 27 is the same as equation 13 for small value of $\zeta$, where equation 3 is reduced to $f \approx g /\left(K^{2} \bar{h}^{1 / 3}\right)$. Using the scaling equation (T2) in Table 1 together with (26) yields the expression for the asymptotic velocity amplitude:

$$
v_{i n f}=\frac{\bar{h}}{a} \frac{c_{0}}{f\left\{8 /(9 \pi)+2 /\left[3 \sqrt{1+\gamma^{2}}\right]\right\}} .
$$

[38] It is interesting to note that if an estuary is long enough, the system will adjust itself until the condition of the ideal estuary is achieved. This is an indication that the ideal estuary is the energetically stable state of an estuary to which the forces of nature converge. Also note that the variables in these equations are all independent variables related to the geometry and the friction, and hence that the asymptotic state is independent of the tidal forcing.

\subsection{Downstream Asymptotic Behavior}

[39] Near the estuary mouth, we can also look at the asymptotic behavior. To what extent is the damping exponential? We can approach the longitudinal damping/amplification of the tidal amplitude by a Taylor series:

$$
\eta^{*} \approx \eta_{0}^{*}+\frac{\mathrm{d} \eta_{0}^{*}}{\mathrm{~d} x} x+\frac{\mathrm{d}^{2} \eta_{0}^{*}}{\mathrm{~d} x^{2}} \frac{x^{2}}{2}+\ldots
$$

[40] On the basis of (20) we can determine the second derivative of $\eta^{*}$ :

$$
\frac{\mathrm{d}^{2} \eta^{*}}{\mathrm{~d} x^{2}}=\frac{1}{(\psi a)^{2}}\left[2 a^{2} \beta^{2} \eta^{* 3}-3 a \beta \eta^{* 2}+\eta^{*}\right] .
$$

[41] Substitution of (20) and (31) into (30) with $\eta_{0}^{*}=1$ yields :

$$
\eta^{*} \approx 1+(1-a \beta) \frac{x}{\psi a}+\frac{1}{2}\left(2 a^{2} \beta^{2}-3 a \beta+1\right)\left(\frac{x}{\psi a}\right)^{2}+\ldots
$$

[42] In a region close enough to the mouth where $x<\psi a$, we can see that the damping or amplification behaves as a linear function of $x$. If $a \beta$ is very small, then

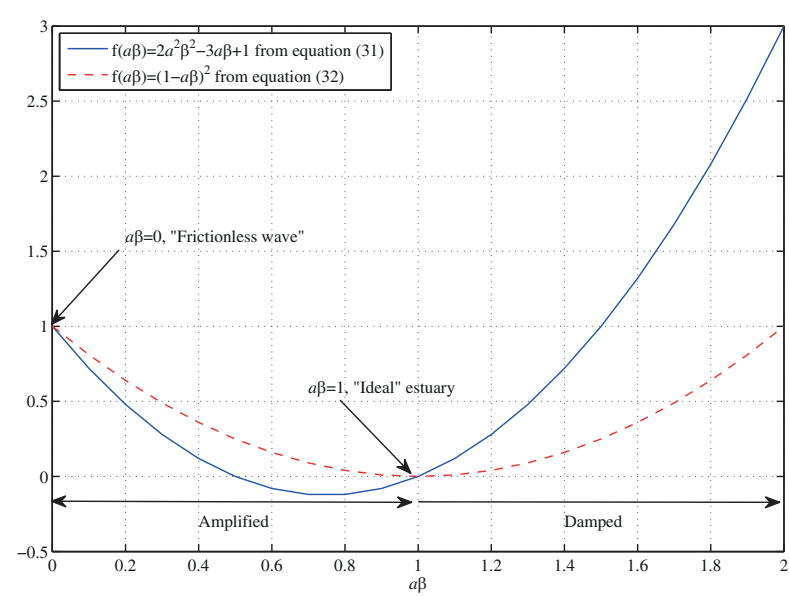

Figure 6. Comparison of the Taylor expansion between the new (32) and the classical (33) damping equations.

the slope is $1 /(\psi a)$. For large values of $a \beta$, the gradient becomes negative and the steeper it gets, the less linear the behavior. A small value of $a \beta$ occurs in deep and strongly converging estuaries, which are generally amplified. Hence, we see that amplification is often linear, as is the case in the Scheldt [Savenije, 2001]. The region where amplification is linear may extend over quite some distance into the estuary. The nonlinear effect only becomes apparent when we move further upstream. In contrast, we see that the process of damping is never linear but closer to an exponential function.

[43] It is interesting to compare the above Taylor series with the Taylor series of the classical exponential damping equation 4. We can develop the exponential equation in a Taylor series as well (making use of (20)):

$$
\eta^{*} \approx 1+(1-a \beta) \frac{x}{\psi a}+\frac{1}{2}(1-a \beta)^{2}\left(\frac{x}{\psi a}\right)^{2}+\ldots
$$

[44] We can then see that the first two terms are the same, but that the higher-order terms are different. Figure 6 compares the factors of the second-order terms. We can see from Figure 6 that the two expressions are only the same in two situations: the ideal estuary (where $a \beta=1$ ) and the frictionless wave (where $a \beta=0$ ). The first case is trivial, because in the ideal case there is no damping, while the second case is an unrealistic case. As a result, the exponential damping assumption is only acceptable if the estuary is near ideal (almost no damping or amplification), or has very low friction. In the next section, we shall show that in real estuaries the use of the classical equation can lead to substantial errors.

\section{Results}

\subsection{Accounting for Local Variability}

[45] The depth, celerity, phase lag, and friction of an estuary are seldom considered constant along the estuary axis. To follow along-channel variations of the estuary geometry or friction, we can split the channel into a series of reaches with different (but constant) friction, phase lag, celerity, depth, etc. [e.g., Souza and Hill, 2006; Savenije 
Table 3. Parameters Used for Analytical Models in the Scheldt Estuary (1955-2006)

\begin{tabular}{|c|c|c|c|c|c|}
\hline Reach (km) & $\begin{array}{l}\text { Convergence } \\
\text { Length, } a(\mathrm{~km})\end{array}$ & $\begin{array}{c}\text { Averaged } \\
\text { Depth, } h(\mathrm{~m})\end{array}$ & $\begin{array}{l}\text { Tidal Amplitude at } \\
\text { Vlissingen, } \eta_{0}(\mathrm{~m})\end{array}$ & $\begin{array}{l}\text { Storage Width } \\
\text { Ratio, } r_{S}\end{array}$ & $\begin{array}{c}\text { Manning-Strickler } \\
\text { Coefficient, } K\left(\mathrm{~m}^{1 / 3} \mathrm{~s}^{-1}\right)\end{array}$ \\
\hline $0-90$ & 27 & $10.5-12.3$ & $1.87-1.96$ & $1.5-1.9$ & 39 \\
\hline $90-180$ & 18 & $5.4-6$ & & $1.3-1.5$ & 20 \\
\hline
\end{tabular}

et al., 2008; Toffolon and Savenije, 2011; Cai et al., 2012a]. Since we derived the explicitly analytical solution for tidal propagation based on only the seaward boundary condition, it can be readily applied in a multireach model moving the origin of the axis for every reach.

[46] For given geometry, friction and tidal amplitude at the seaward boundary $\eta_{0}$, we are able to compute the shape number $\gamma$ and the friction number at the estuary mouth $\chi_{0}$. We thereby assume constant values of $\mu, \lambda, \varepsilon$ within each reach (calculated at the origin of each reach), which can be computed by solving the set of equations in Cai et al. [2012a]. Unlike Cai et al. [2012a] who solved the longitudinal tidal amplitude by numerical integration of the damping number $\delta$ over a length step $\Delta x$, we calculate the tidal amplitude variation along the estuary axis directly from the new equation 22. The use of the explicit equation 22 leads to an updated tidal amplitude $\eta_{1}$ (hence friction number $\chi_{1}$ ) at a distance interval $\Delta x$ (e.g., $1 \mathrm{~km}$ ) upstream, which is repeated for the whole estuary.

[47] For the case of negligible river discharge, it is customary that classical studies [e.g., Hunt, 1964; Ippen, 1966; Friedrichs and Aubrey, 1994; Friedrichs, 2010; Van Rijn, 2011] use the exponential function (4) to describe the longitudinal variation of the tidal amplitude along the estuary axis. It is important to note that in these studies the damping number, the celerity number, and the phase lag are assumed to be constant for the entire estuary reach. The two undetermined parameters (i.e., the Manning-Strickler friction coefficient $K$ and the storage width ratio $r_{S}$ ) are subsequently calibrated by comparing the computed results against observations of tidal amplitude and travel time of the tidal wave. While the damping/amplification is sensitive to both friction and storage width ratio, the wave celerity is specifically sensitive to the $r_{S}$. The method presented here differs from these earlier studies in that we derive local solutions depending on the local tidal amplitude to depth ratio $\zeta$, which enables the model to take account of along-channel variations of the estuary geometry (e.g., the depth and the storage width ratio) or the friction. The whole estuary can be divided into multiple reaches of length $\Delta x$ (e.g., $1 \mathrm{~km}$ ) with constant depth and friction while the variable tidal amplitude is obtained by the explicit equation 22 . The same method can be applied using the classical linear solutions [see, for instance, Toffolon and Savenije, 2011].

[48] Incorporating the new explicit equation 22 into a multireach approach with $\Delta x=1 \mathrm{~km}$, the Hybrid model [Cai et al., 2012a] has been applied to the Scheldt estuary. The total length of the estuary is about $180 \mathrm{~km}$ from the estuary mouth at Vlissingen to the estuary head near Gent (closed by a weir). The annual observations of tidal amplitude and travel time at HW and LW along the Scheldt between 1955 and 2006 have been used to calibrate and verify the model. The cross-sectional area of the estuary can be well represented by an exponential function (1) with a convergence length $a=27 \mathrm{~km}$ in the seaward part (0-90 $\mathrm{km})$ and $a=18 \mathrm{~km}(90-180 \mathrm{~km})$ in the upstream part [see also Horrevoets et al., 2004]. The reduction of the convergence length is due to the shallowing, whereby the upstream part has experienced less dredging. Until $90 \mathrm{~km}$ from the estuary mouth ( $x=0 \mathrm{~km}$, Vlissingen $)$ it is observed that the flow depth is approximately constant, while more landward the depth gradually reduces (until about $3 \mathrm{~m}$ ) as the estuary becomes more riverine in character. Table 3 presents the geometric and flow characteristics as well as the calibrated parameters, including the storage width ratio $r_{S}$ and Manning-Strickler coefficient $K$ on which the computation is based. During the examined period (1955-2006), according to the cross-sectional survey, the annually averaged depth of the seaward part $(x=0-90 \mathrm{~km})$ was deepened from 10.5 to $12.3 \mathrm{~m}$.

[49] In Figures 7 and 8, the analytically computed tidal amplitudes are compared with the observations along the Scheldt estuary. We can see that the correspondence with observed values is good, both in the seaward part $(x=0-90$ $\mathrm{km}$ ) where the depth is close to constant and in the landward part $(x=90-180 \mathrm{~km})$ where the depth gradually reduces. The model fits the observations with constant values of Manning-Strickler coefficient, i.e., $39 \mathrm{~m}^{1 / 3} \mathrm{~s}^{-1}$ in the seaward part and $20 \mathrm{~m}^{1 / 3} \mathrm{~s}^{-1}$ in the landward part, respectively (see Table 3). It is worth noting that the calibrated friction coefficient in the upstream part is rather small $\left(K=20 \mathrm{~m}^{1 / 3} \mathrm{~s}^{-1}\right.$, hence big friction), which is due to the neglect of river discharge in the equations. The neglect of river discharge can be compensated by increasing the friction [Cai et al., 2012b]. Further work will be needed to include the effect of river discharge in this model.

[50] To demonstrate the practical importance of the proposed multireach method, Figure 9 compares the performance of two analytical models (both with fine (\#1, \#2) and coarse (\#3, \#4) discretization) applied to the Scheldt estuary, compared against annual observations of tidal amplitude and travel time for HW and LW in 2000 (the Matlab scripts are provided as supporting information). The models \#1 and \#2 use a multireach approach with a small length step $\Delta x=1 \mathrm{~km}, \# 1$ adopting the explicit equation 22 and $\# 2$ the exponential damping equation 4 for the tidal amplitude, respectively. Models \#3 and \#4 use a large length step (i.e., $90 \mathrm{~km}$ in the seaward part $x=0-90 \mathrm{~km}$ and $30 \mathrm{~km}$ in the upstream part $x=90-180 \mathrm{~km}$ ), making use of the explicit equation 22 and the exponential damping equation 4 , respectively. The damping number $\delta$ in the exponential equation 4 was estimated by the hybrid model of Cai et al. [2012a]. All models use the same roughness values as presented in Table 3. It can be seen from Figure 9 that the performance of model \#1 and model \#2 is almost identical for small length step, which indicates that equation 4 is a good 

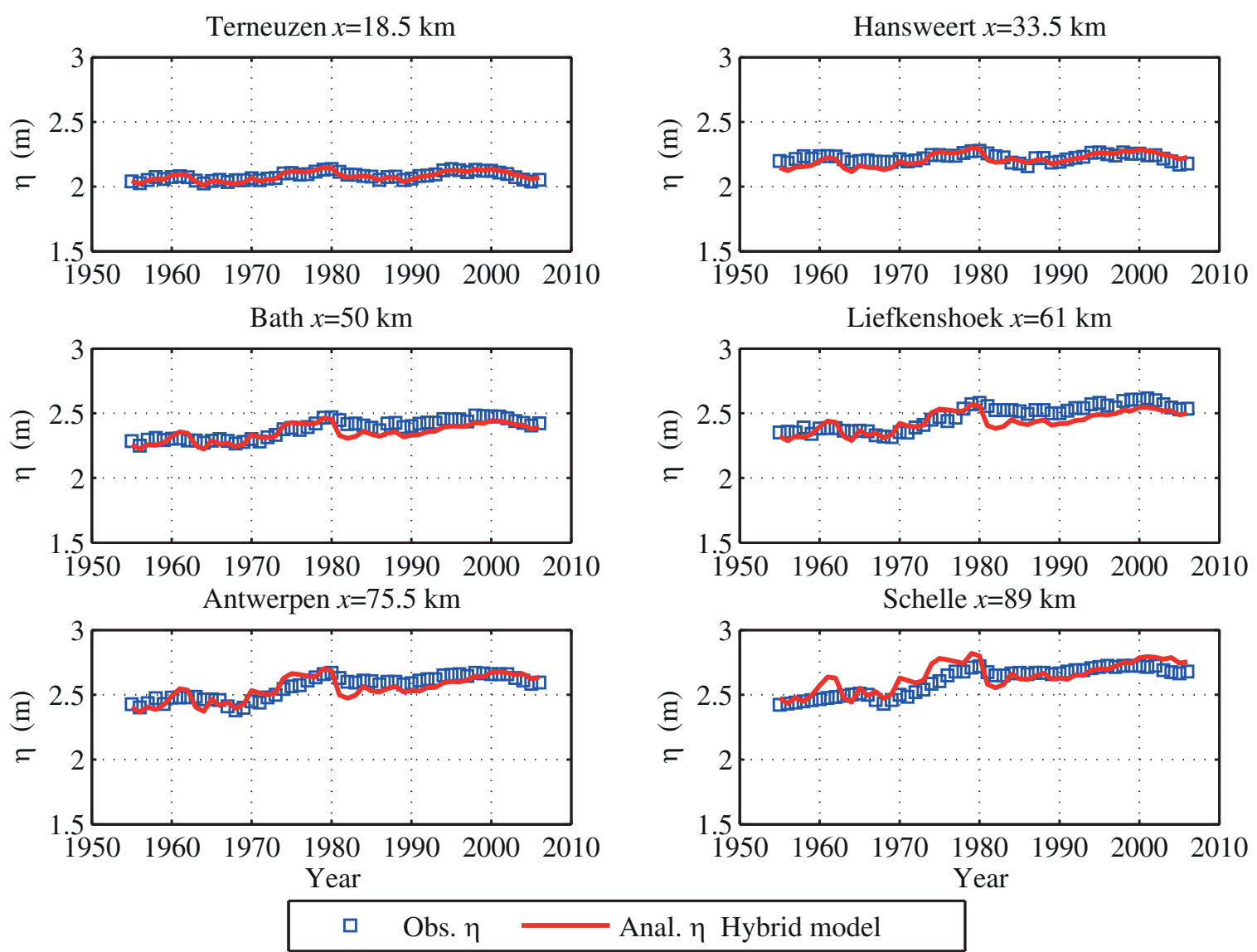

Figure 7. Comparison between the analytically computed tidal amplitude and measurements in the seaward part (0-90 km) of the Scheldt estuary at different locations.

first-order approximation of the proposed explicit equation 22 as long as $\Delta x$ is small. For the model of \#3, the correspondence with observed tidal amplitude is surprisingly good even using large length steps. The reason why the fit is not perfect is because in \#3 $f$ is rather constant, whereas it is $\zeta$ dependent to cater for the tidal depth variability in the denominator of the friction term. In model \#3 we have to assume that $f$ remains constant over a reach. Model \#4 is less accurate for larger length steps. This is because the new method has asymptotic values both for amplified and damped conditions, whereas the classical method tends to infinity for an amplified wave and to zero for a damped wave. For the travel times, the methods do not differ much. It is worth noting that model \#4 can be made to fit observations, but only by adjusting the roughness values for the different reaches. It would have required a larger roughness coefficient in the downstream part and a smaller roughness in the upstream part to fit the observations.

\subsection{Overamplification Induced by Deepening}

[51] The tidal amplitude along the Scheldt estuary can be well simulated by the analytical model, which suggests that the analytical solution presented in this paper is a very powerful instrument to assess the possible influence of human interference in the estuarine system, such as dredging and deepening of navigation channels. During the past century, the navigation channel to the Port of Antwerp in Belgium was deepened several times and at present it is maintained by annual dredging. Due to the effect of depth increase, the tidal amplitude and wave celerity have greatly increased over the last half century. However, tidal amplification is not a straightforward function of depth. There appears to be a critical depth, which causes maximum amplification, beyond which the amplification is reduced as the wave gradually assumes the properties of a standing wave. To minimize the environmental impacts of tidal wave amplification, we need to fully understand the nonlinear relationship between deepening and tidal amplification. The new analytical equation 22 is an excellent tool for this. Since they provide direct insight into the threshold, the asymptotic value, and the functional relationship that governs amplification.

[52] To evaluate the effect of deepening on tidal dynamics in the Scheldt estuary, various computations have been made under different depths (ranging from 5 to $25 \mathrm{~m}$ ) using the proposed hybrid analytical model and the 1-D numerical model (as described in Toffolon et al. [2006]) with the same tidal amplitude at the seaward boundary $(1.9 \mathrm{~m}$ corresponding with the annual average tidal amplitude between 1955 and 2006) and fixed storage width ratio of 1.6 (estimated by fitting the average annual tidal observations during 1955-2006). Figure 10 shows the effect of deepening on the tidal damping/amplification ratio $\left(\eta / \eta_{0}\right)$ at different locations along the primary navigation channel $(0-90 \mathrm{~km})$. We can see that the analytically computed values are in 

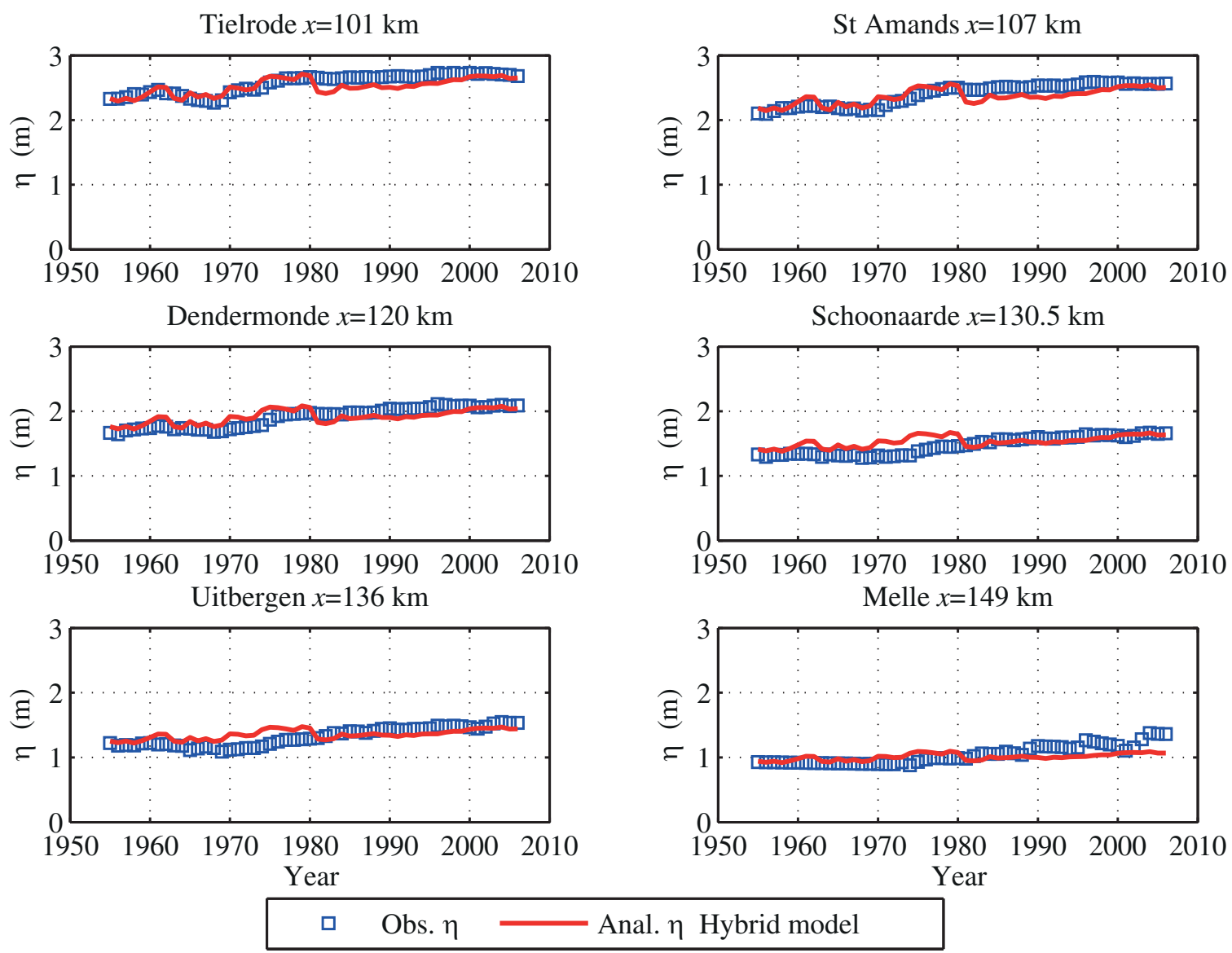

Figure 8. Comparison between the analytically computed tidal amplitude and measurements in the upstream part (90-180 km) of the Scheldt estuary at different locations.

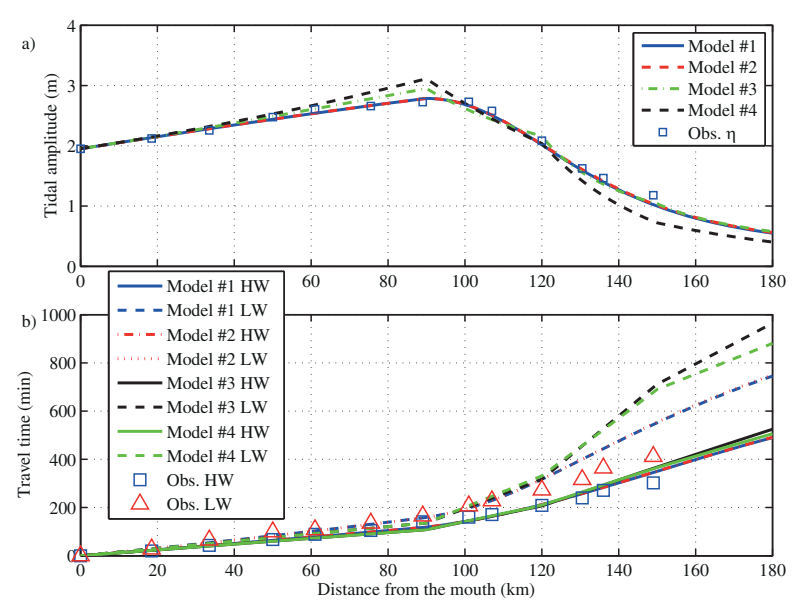

Figure 9. Comparison between different analytical models and observations: (a) tidal amplitude and (b) travel time at HW and LW in the Scheldt estuary observed in 2000. Models \#1 (using equation 22) and \#2 (using equation 4) make use of multireach approach with small length step $\Delta x=1 \mathrm{~km}$, while models \#3 (using equation 22) and \#4 (using (4)) use large length steps $\Delta x=90 \mathrm{~km}$ (in the seaward part $x=0-90 \mathrm{~km}$ ) and $30 \mathrm{~km}$ (in the landward part $x=90-180 \mathrm{~km})$. good agreement with the measured tidal amplification ratio at the different stations. The results of the numerical model are also shown in Figure 10. In general, the correspondence between the analytical model and the numerical model is good, although the analytical results are slightly overestimated for averaged depth between 12 and $18 \mathrm{~m}$ and more so in the upstream part. This is due to the deformation of the wave which the analytical model does not consider. The analytical model shows that tidal damping $\left(\eta / \eta_{0}<1\right)$ occurs for a depth smaller than about $7 \mathrm{~m}$ due to the dominant effect of friction, while the wave becomes amplified $\left(\eta / \eta_{0}>1\right)$ for a depth larger than $7 \mathrm{~m}$ when the convergence is stronger than friction. We can demonstrate that a depth increase only leads to increased amplification (larger $\left.\eta / \eta_{0}\right)$ until a maximum value is reached at a critical depth $\bar{h}_{\text {critical }}$ defined by the condition

$$
\frac{\partial \eta}{\partial \bar{h}}=0
$$

[53] For the quasi-nonlinear model, this condition for maximum amplification is similar to the one defined by Savenije et al. [2008] as the threshold for the critical convergence at which the tide switches from mixed wave to the "apparently standing" wave (the wave is not a formally standing wave generated by the superimposition of 

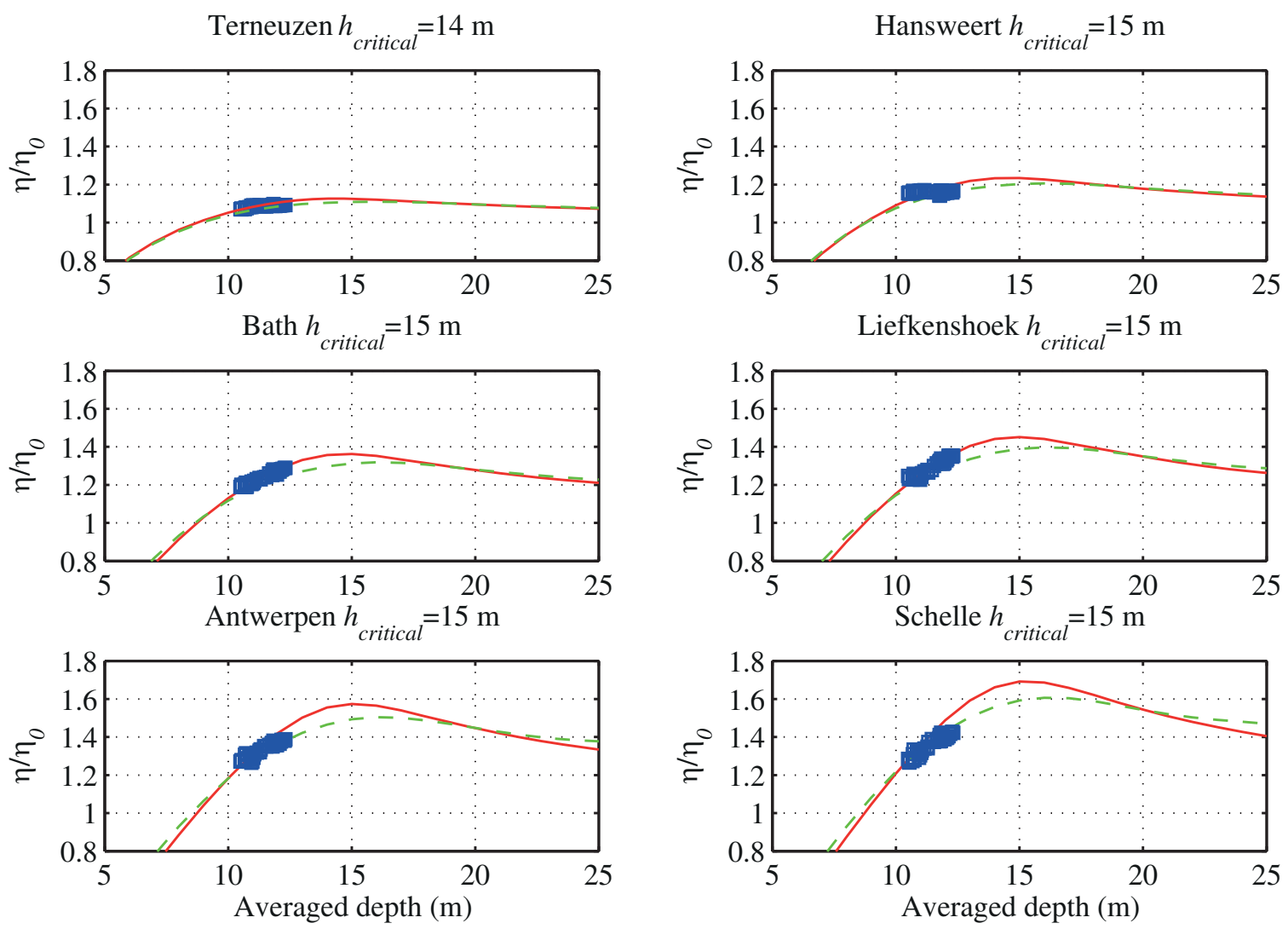

O Obs. $\eta / \eta_{0} \longrightarrow$ Anal. $\eta / \eta_{0}---$ Num. $\eta / \eta_{0}$

Figure 10. Observed tidal amplification ratio as function of averaged depth along the primary navigation channel in the Scheldt estuary, compared to the amplification computed with the hybrid model (continuous lines) and the 1-D numerical model (dashed lines).

incident and reflected waves; rather it is an incident wave that mimics a standing wave having a phase difference of $90^{\circ}$ between water level and velocity and a wave celerity tending to infinity). Rewriting their equation (44a) for the critical shape number $\gamma_{c}$ as a function of $\bar{h}_{\text {critical leads to: }}$

$$
\begin{gathered}
\bar{h}_{\text {critical }}=r_{S} \gamma_{c}^{2} \omega^{2} a^{2} / g, \quad \gamma_{c}=\frac{1}{3 \chi}\left(m_{1} / 2-1+\left(24 \chi^{2}+2\right) / m_{1}\right), \\
m_{1}=\left[108 \chi^{4}+288 \chi^{2}-8+\sqrt{432 \chi^{2}\left(\chi^{2}-2\right)^{2}\left(27 \chi^{2}-4\right)}\right]^{1 / 3} .
\end{gathered}
$$

[54] This value based on critical convergence rather than on the critical depth defined by (34) provides a slightly smaller value of $\bar{h}_{\text {critical }}$. It is worth noting that the system flips suddenly to a frictionless standing wave after reaching maximum amplification (i.e., $\bar{h} \geq \bar{h}_{\text {critical }}$ ) due to the discontinuous transition to a standing wave predicted by Savenije et al. [2008].

[55] It is worth noting that the critical convergence defined by Jay [1991] is the rate at which the topographic convergence is balanced by the effect of acceleration, where the estuary shape number $\gamma=2$, which is the same value as obtained by our hybrid model for a frictionless wave system.
[56] The tidal amplification ratio reduces for a depth larger than the critical depth, i.e., about $15 \mathrm{~m}$ for the main navigational channel. And it can be seen from Figure 10 that the maximum amplification increases in landward direction, from about 1.1 in Terneuzen to 1.7 in Schelle. Cai et al. [2012a] classified estuaries having a depth $\bar{h}>$ $\bar{h}_{\text {critical }}$ as "overamplified," where increasing the depth reduces the tidal amplification. The same phenomenon of "overamplification" was observed by Van Rijn [2011] using an energy-based method, which in fact is identical to the linear solution. Instead of using a multireach implementation (as described by section 5.1), Van Rijn [2011] obtained the longitudinal tidal amplitude by applying the exponential equation 4.

[57] Since the hybrid model consists of four implicit equations (see Table 1), the reaction of tidal wave propagation to the deepening cannot be observed directly from these equations. To illustrate the effect of deepening in the Scheldt estuary $(0-90 \mathrm{~km})$, we present the trajectory of the four main dimensionless parameters as function of averaged depth $(5 \leq \bar{h} \leq 25 \mathrm{~m})$ in Figure 11 . We can see that the velocity number and the damping number increase until a maximum value is reached. A further increase of the depth reduces the tidal amplification (both tidal amplitude and velocity amplitude) until critical convergence is reached asymptotically, where the celerity number and the 

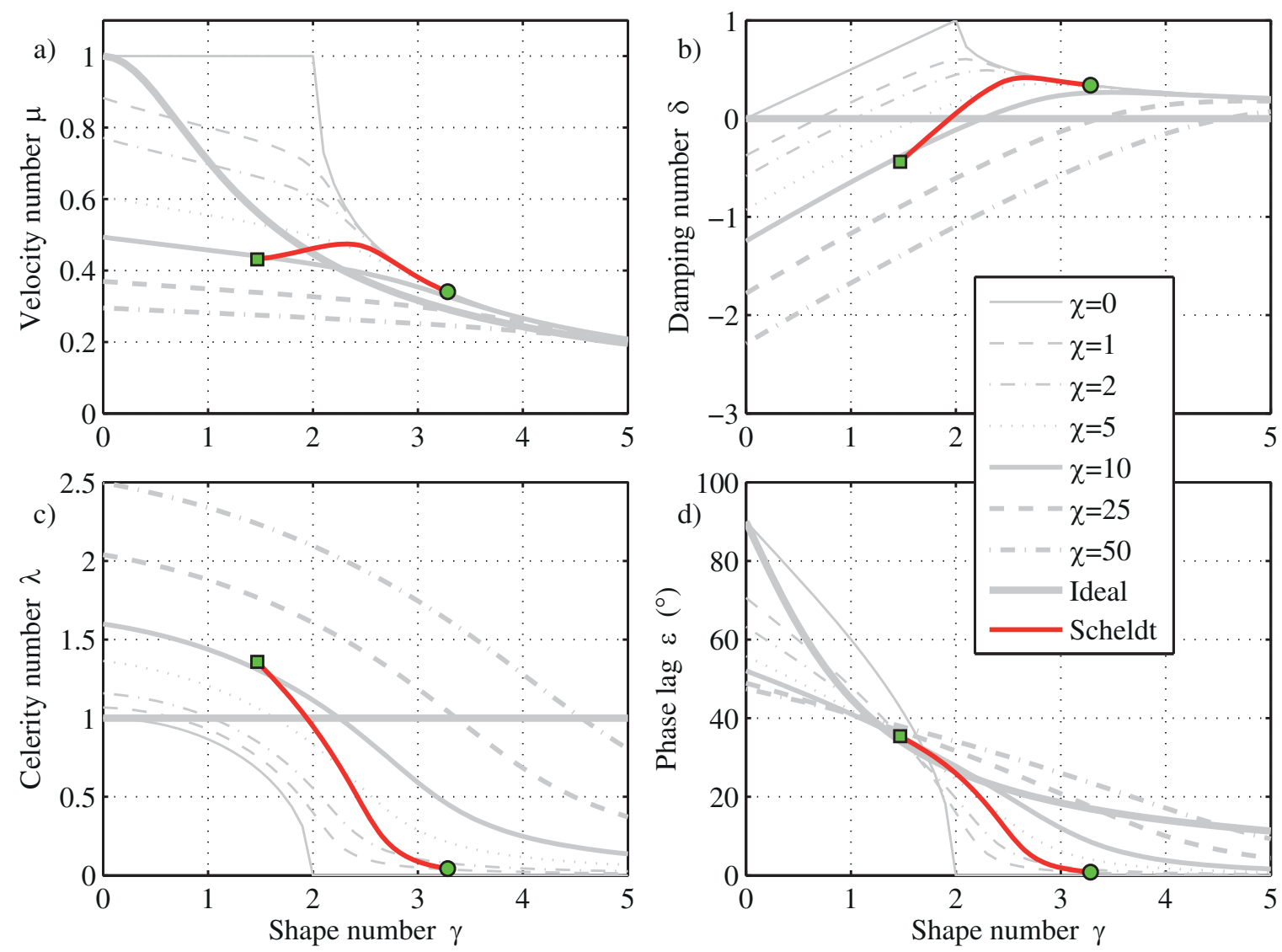

Figure 11. Trajectories of the main dimensionless parameters as function of averaged depth in the Scheldt estuary (0-90 km, red segments) in: (a) velocity number diagram, (b) damping number diagram, (c) celerity number diagram, and (d) phase lag diagram. The green square symbols indicate the initial position with averaged depth of $5 \mathrm{~m}$, while the green circle symbols represent the final position with averaged depth of $25 \mathrm{~m}$. The background shows the lines of the hybrid model with different values of the friction number $\chi$.

phase lag approach zero (see Figures 11c and 11d), corresponding with a frictionless standing wave system. For the case of critical convergence, the solutions are identical to those obtained by the Savenije et al. [2008] for the second family of solution ("apparently standing" wave) and the solutions are completely determined by the convergence alone, i.e.,

$$
\lambda=\varepsilon=0, \quad \mu=\delta=\left(\gamma-\sqrt{\gamma^{2}-4}\right) / 2 .
$$

[58] When the estuary shape number $\gamma$ goes to infinity (e.g., $\bar{h}$ approaches infinity), we can see that both $\mu$ and $\delta$ approach zero asymptotically. From a physical point of view, we can derive from the dimensional damping equation 16 that if depth is increased (hence friction becomes smaller), the friction term on the right-hand side, i.e., $2 f v[4 /(3 \pi)+\sin (\varepsilon)] /(3 \bar{h} c)$, becomes smaller, leading to more amplification. However, the term contained in the parenthesis on the left-hand side, i.e., $1+g \eta /(\operatorname{cosin}(\varepsilon))=1+1 / \mu^{2}$, becomes larger with increasing depth, leading to less amplification. The term $1 / \mu^{2}$ reflects the ratio of gravity to acceleration and only becomes dominant for small values of $\mu$. The maximum amplification stems from the trade off between these terms.
[59] In Figure 12, we present the analytical values of the velocity amplitude and tidal amplitude as well as their corresponding dimensionless numbers (velocity number and
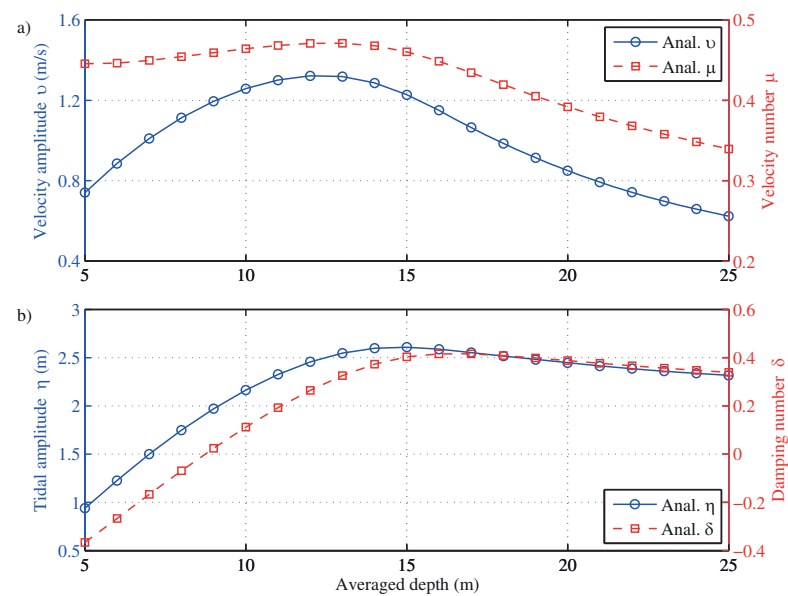

Figure 12. (a) The velocity amplitude as well as the velocity number and (b) the tidal amplitude along with the damping number as function of averaged depth at Bath $(x=50 \mathrm{~km})$ in the Scheldt estuary. 
CAI AND SAVENIJE: ASYMPTOTIC BEHAVIOR

Table 4. Characteristic Values of Alluvial Estuaries and Classification

\begin{tabular}{|c|c|c|c|c|c|c|c|c|c|c|c|c|}
\hline Number & Estuary $^{\mathrm{a}}$ & $T(\mathrm{~h})$ & $\eta_{0}(\mathrm{~m})$ & $\bar{h}(\mathrm{~m})$ & $a(\mathrm{~km})$ & $K\left(\mathrm{~m}^{1 / 3} \mathrm{~s}^{-1}\right)$ & $\zeta$ & $\gamma$ & $a \beta$ & $\eta_{\text {inf }}(\mathrm{m})$ & $v_{\text {inf }}(\mathrm{m} / \mathrm{s})$ & Type \\
\hline 1 & Bristol Channel & 12.4 & 2.6 & 45 & 65 & 33 & 0.06 & 2.3 & 0.1 & 25.09 & 12.16 & Amplified \\
\hline 2 & Columbia & 12.4 & 1 & 10 & 25 & 38 & 0.1 & 2.81 & 0.22 & 4.63 & 1.54 & Amplified \\
\hline 4 & Elbe & 12.4 & 2 & 10 & 42 & 43 & 0.2 & 1.68 & 0.76 & 2.64 & 2.68 & Amplified \\
\hline 5 & Fraser & 12.4 & 1.5 & 9 & 215 & 31 & 0.17 & 0.31 & 17.16 & 0.09 & 0.13 & Damped \\
\hline 6 & Gironde & 12.4 & 2.3 & 10 & 44 & 38 & 0.23 & 1.6 & 1.16 & 1.99 & 2.4 & Close to ideal \\
\hline 8 & Ord & 12 & 2.5 & 4 & 15.2 & 50 & 0.63 & 2.83 & 1.46 & 1.71 & 2.23 & Damped \\
\hline 9 & Outer Bay of Fundy & 12.4 & 2.1 & 60 & 230 & 33 & 0.04 & 0.75 & 0.21 & 9.91 & 6.73 & Amplified \\
\hline 10 & Potomac & 12.4 & 0.65 & 6 & 54 & 56 & 0.11 & 1.01 & 0.91 & 0.71 & 0.42 & Close to ideal \\
\hline 11 & Scheldt & 12.4 & 1.9 & 11 & 27 & 39 & 0.17 & 2.16 & 0.56 & 3.39 & 3.23 & Amplified \\
\hline 12 & Severn & 12.4 & 3 & 15 & 41 & 40 & 0.2 & 2.1 & 0.48 & 6.24 & 6.51 & Amplified \\
\hline 13 & St. Lawrence & 12.4 & 2.5 & 70 & 183 & 44 & 0.04 & 1.02 & 0.09 & 28.88 & 18.95 & Amplified \\
\hline 14 & Tees & 12 & 1.5 & 7.5 & 5.5 & 36 & 0.2 & 10.72 & 0.28 & 5.44 & 0.87 & Amplified \\
\hline 17 & Pungue & 12.4 & 3 & 4.3 & 20 & 33 & 0.7 & 2.11 & 5.42 & 0.55 & 1.18 & Damped \\
\hline 18 & Lalang & 12.4 & 1.5 & 10.6 & 217 & 70 & 0.14 & 0.33 & 2.31 & 0.65 & 0.89 & Damped \\
\hline 19 & Tha Chin & 12.4 & 1.2 & 5.3 & 87 & 50 & 0.23 & 0.56 & 6.95 & 0.17 & 0.26 & Damped \\
\hline 20 & Incomati & 12.4 & 1.35 & 3 & 42 & 63 & 0.45 & 0.75 & 10.62 & 0.13 & 0.3 & Damped \\
\hline 21 & Limpopo & 12.4 & 0.55 & 7 & 50 & 43 & 0.08 & 1.18 & 0.73 & 0.75 & 0.32 & Amplified \\
\hline 22 & Maputo & 12.4 & 1.4 & 3.6 & 16 & 70 & 0.39 & 2.41 & 0.87 & 1.61 & 1.56 & Close to ideal \\
\hline 23 & Chao Phya & 12.4 & 0.9 & 8 & 109 & 51 & 0.11 & 0.51 & 2.95 & 0.31 & 0.31 & Damped \\
\hline
\end{tabular}

${ }^{\mathrm{a}}$ Data are modified from Cai et al. [2012a].
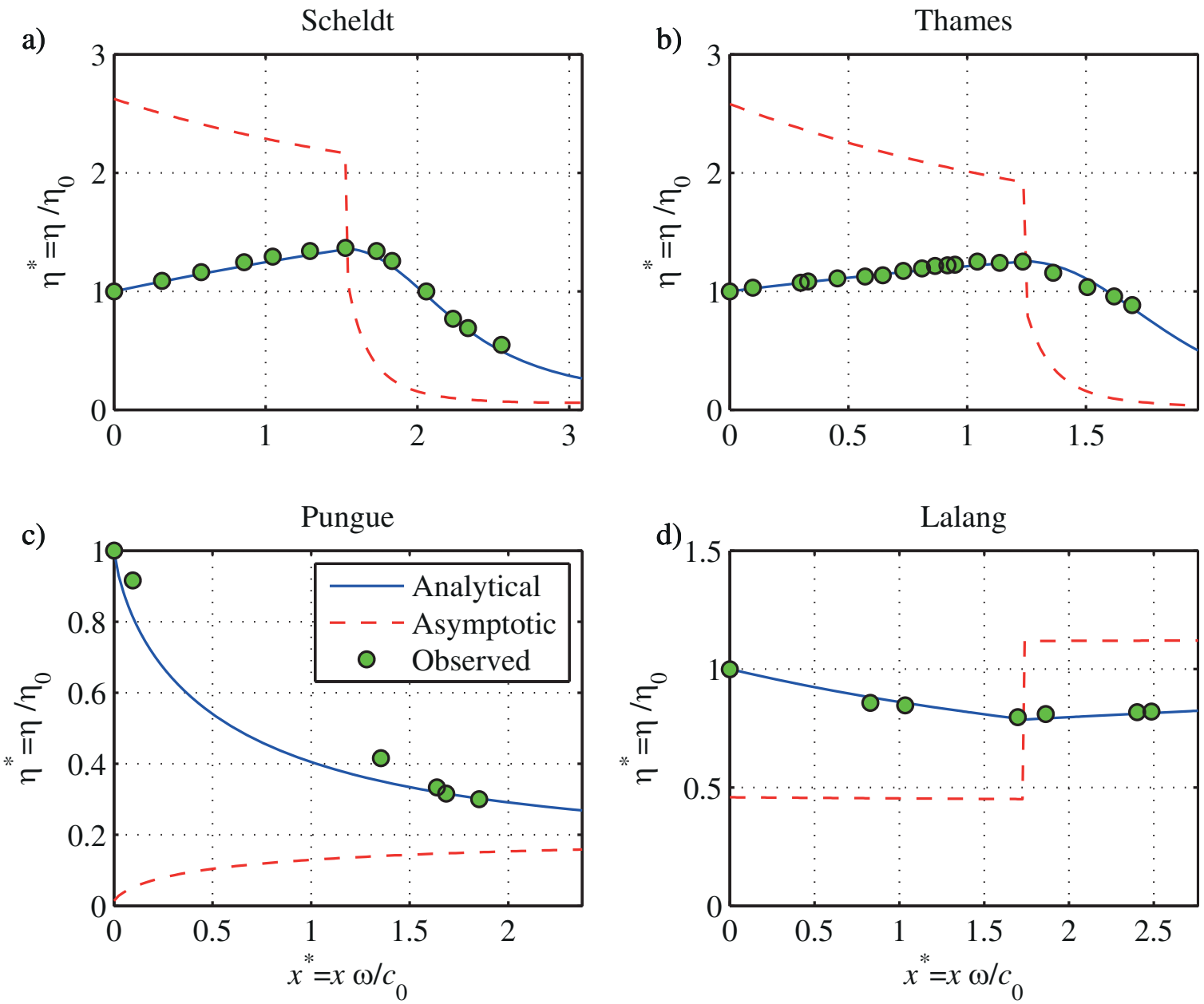

Figure 13. Observed and computed longitudinal variation of tidal amplitude in selected estuaries ((a) Scheldt; (b) Thames; (c) Pungue; (d) Lalang). The red dashed line represents the asymptotic tidal amplitude obtained with equation 27 as a function of the locally observed geometry. 

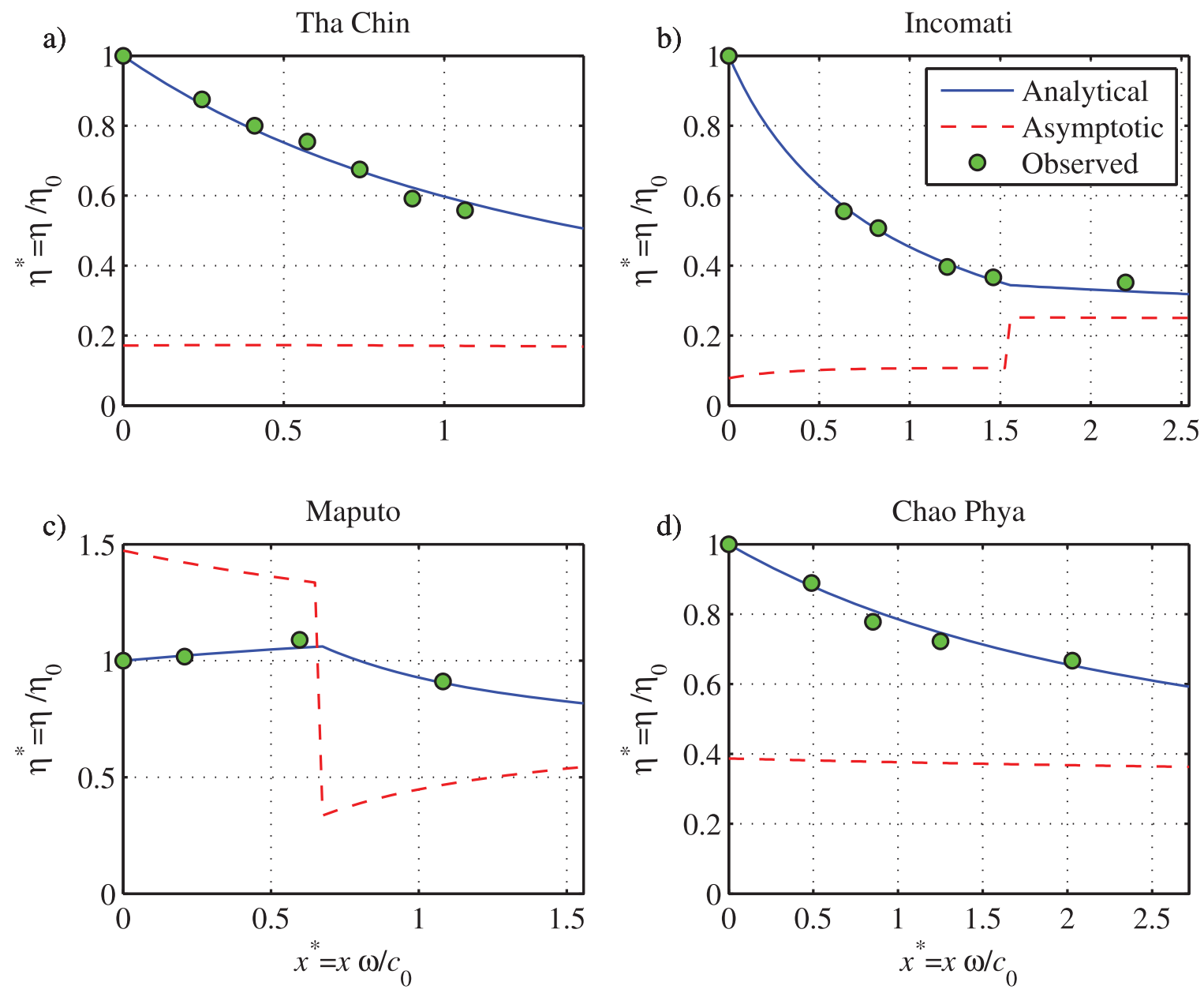

Figure 14. Observed and computed longitudinal variation of tidal amplitude in selected estuaries: (a) Tha Chin; (b) Incomati; (c) Maputo; (d) Chao Phya. The red dashed line represents the asymptotic tidal amplitude obtained with equation 27 as a function of the locally observed geometry.

damping number) as a function of the averaged depth at Bath $(x=50 \mathrm{~km})$. As the depth increases, both the velocity amplitude and tidal amplitude increase until a maximum value is reached at critical depth. The critical depth for the velocity amplitude and the velocity number are about $12 \mathrm{~m}$, while the critical depth for the tidal amplitude is about 15 $\mathrm{m}$, which is slightly smaller than the critical depth of the damping number at $16 \mathrm{~m}$ depth. These differences follow directly from the definitions of $\delta$ and $\mu$ as function of depth (see Table 2).

\subsection{Classification of Estuary}

[60] The asymptotic tidal amplitudes $\eta_{\text {inf }}$ and velocity amplitude $v_{\text {inf }}$ of a selection of estuaries are shown in Table 4 , where $\eta_{\text {inf }}$ is calculated with equation 10 while $v_{\text {inf }}$ is computed with equation 29. Qualitatively, we can determine whether an estuary is amplified or damped by comparing the difference between the tidal amplitude at the estuary mouth $\eta_{0}$ and the asymptotic (or ideal) tidal amplitude $\eta_{\text {inf }}$. For amplified estuaries where $\eta_{0}<\eta_{\text {inf }}(a \beta<1)$, such as Bristol Channel, Columbia, Outer Bay of Fundy, Scheldt, Severn, St. Lawrence, and Tees, a larger asymptotic tidal amplitude $\eta_{\text {inf }}$ and velocity amplitude $v_{\text {inf }}$ is obtained. For damped estuaries where $\eta_{0}>\eta_{\text {inf }}(a \beta>1)$, like Fraser, Ord, Gambia, Pungue, Lalang, Tha Chin, Incomati, and Chao Phya, we can see $\eta_{\text {inf }}$ and $v_{\text {inf }}$ achieve a lower asymptotic value. We can also see that the Gironde, Hudson, Potomac, and Maputo are very close to ideal estuaries with $\eta_{\text {inf }} \approx \eta_{0}(a \beta \approx 1)$.

\subsection{Application to Real Estuaries}

[61] Figures 13 and 14 show longitudinal computations applied to the Scheldt, Thames, Pungue, Lalang, Tha Chin, Incomati, Maputo, and Chao Phya estuaries where tidal damping observations were available. Details on the geometric parameters used for these calculation are shown in the supporting information. It can be seen from Figures 13 and 14 that the model fits the observed tidal amplitude very well and the estimated asymptotic tidal amplitude (27) is in good agreement with the observations. The fact that the Lalang shows larger amplitude upstream is due to an increasing depth in the upstream direction [Savenije, 1992b]. On the other hand, the strong upstream damping in the Scheldt and Thames is due to upstream shallowing. The jump in the asymptotic value near $x^{*}=1.7$ (Scheldt) and $x^{*}=1.3$ (Thames) is due to the change of the convergence length, which generates an almost standing wave over a 
short distance before the depth reduction further upstream again reduces the asymptotic amplitude.

[62] It is worth noting that the proposed hybrid analytical model is able to accurately reproduce the main tidal hydrodynamics by following along-channel variation of estuarine sections (e.g., the depth). For instance, Figure 13a shows the computed and measured tidal amplitude along the Scheldt estuary, even in the upstream part, where the depth gradually reduces. We can see that the seaward part (0-90 $\mathrm{km}$ ) can be classified as amplified, with a large asymptotic tidal amplitude, while the upstream part $(90-180 \mathrm{~km})$ is significantly damped with a much smaller asymptotic tidal amplitude.

\section{Conclusions}

[63] In this study, we have presented fully explicit expressions for tidal amplitude and velocity amplitude along the estuary axis. The derived equations provide direct insight into the hydrodynamics of estuaries. The solutions depend on two parameters, i.e., the shape number $\gamma$ and the friction number at the estuary mouth $\chi_{0}$. These simple expressions can be easily incorporated in the model proposed by Cai et al. [2012a] with a multireach technique, i.e., subdividing the estuary into multiple reaches. The multireach implementation enables the analytical model to take account of local variability (e.g., the depth or friction) and performs much better than the classical exponential equation 4 applied to the entire estuary (i.e., single reach).

[64] We have compared the performance between the classical linear solution and those proposed by Savenije et al. [2008], Toffolon and Savenije [2011], and Cai et al. [2012a]. It is found that exponential damping is only valid when assuming a constant friction number $\chi$ along the estuary axis, which in fact implies a constant amplitude, and hence an ideal estuary. The more realistic situation is that estuaries converge toward an asymptote where the impact of convergence is balanced by friction.

[65] We have also shown that the upstream asymptotic state is basically independent of tidal forcing, which indicates that an estuary adjusts the tidal amplitude to the estuary shape until it has the same properties as in an ideal estuary with spatially constant parameters. For the downstream asymptotic behavior of the damping equation, it has been shown that damping/amplification is linear in a region close to the estuary mouth, particularly in the case of amplification, such as in the seaward part of the Scheldt estuary. The nonlinear effect only becomes significant in the upper reaches of the estuary. The analysis of the downstream asymptotic behavior of tidal damping demonstrates that the classical exponential damping equation 4 is only valid when there is almost no damping or amplification (which is trivial) or for a frictionless wave (which is unrealistic).

[66] The analytical solutions are compared with half a century of observations in the Scheldt estuary, which was substantially deepened over that period. The correspondence with observations is very good. The analytical model has subsequently been applied to investigate the effect of further deepening on the tidal dynamics. Interestingly, there is a critical depth beyond which the amplifica- tion is reduced until critical convergence (frictionless and standing wave system) is reached asymptotically. Finally, the asymptotic behavior can be used to classify estuaries as damped, amplified, or ideal by comparing the tidal amplitude at the estuary mouth with that of an ideal estuary.

\section{Appendix A: Derivation of the Explicit Solution to the Quasi-Nonlinear Tidal Damping Equation}

[67] Based on the full nonlinear St. Venant equations, Savenije [1998, 2001, 2005] determined an analytical expression for tidal damping by subtracting high water (HW) and low water (LW) envelopes:

$$
\frac{1}{\eta} \frac{\mathrm{d} \eta}{\mathrm{d} x}\left[1+\frac{g \eta}{c v \sin (\varepsilon)}\right]=\frac{1}{a}-f \frac{v \sin (\varepsilon)}{\bar{h} c},
$$

[68] Using the same assumptions made in section 3 and introducing a new parameter

$$
\beta_{Q}=\frac{f v_{0} \sin (\varepsilon)}{\bar{h} c}
$$

equation (A1) can be simplified as:

$$
\frac{\mathrm{d} \eta^{*}}{\mathrm{~d} x}=\frac{\eta^{*}}{\psi a}\left(1-a \beta_{Q} \eta^{*}\right) .
$$

[69] Equation (A3) can be integrated by separation of variables. Applying the boundary condition $\eta^{*}=1$ at $x=0$, integration yields an explicit solution for tidal damping:

$$
\begin{aligned}
\eta^{*} & =\frac{1}{a \beta_{Q}+\left(1-a \beta_{Q}\right) \exp [-x /(\psi a)]} \\
& =\frac{1 /\left(a \beta_{Q}\right)}{1-\left[1-1 /\left(a \beta_{Q}\right)\right] \exp [-x /(\psi a)]} .
\end{aligned}
$$

[70] Introducing the dimensionless parameters defined in Table 1, equation (A4) can be rewritten as:

$$
\eta^{*}=\frac{\gamma /\left(\chi_{0} \mu_{0}^{2} \lambda^{2}\right)}{1-\left[1-\gamma /\left(\chi_{0} \mu_{0}^{2} \lambda^{2}\right)\right] \exp \left[-\gamma \mu_{0}^{2} x^{*} /\left(1+\mu_{0}^{2}\right)\right]},
$$

which gives the asymptotic tidal amplitude $\eta_{\text {inf }}^{*}=\gamma /\left(\chi_{0} \mu_{0}^{2} \lambda^{2}\right)$ when $\chi^{*}$ goes to infinity.

\section{Appendix B : Derivation of the Explicit Solution to the Linear Tidal Damping Equation}

[71] Cai et al. [2012a] adopted the envelope method using the usual Lorentz's linearization for the friction term and derived the linear tidal damping equation:

$$
\frac{1}{\eta} \frac{\mathrm{d} \eta}{\mathrm{d} x}\left[1+\frac{g \eta}{\operatorname{cosin}(\varepsilon)}\right]=\frac{1}{a}-\frac{8}{3 \pi} f \frac{v}{\bar{h} c}
$$

[72] Following the derivation as described in Appendix A, one can easily obtain the following explicit solution of the linear tidal damping equation: 


$$
\eta^{*}=\frac{3 \pi \gamma /\left(8 \chi_{0} \mu_{0} \lambda\right)}{1-\left[1-3 \pi \gamma /\left(8 \chi_{0} \mu \lambda\right)\right] \exp \left[-\gamma \mu^{2} x^{*} /\left(1+\mu_{0}^{2}\right)\right]},
$$

[73] We can see from equation (B2) that $\eta_{\text {inf }}^{*}=3 \pi \gamma /$ $\left(8 \chi_{0} \mu \lambda\right)$ when $x^{*}$ approaches infinity.

\section{Notation}

The following symbols are used in this paper (asterisk denotes dimensionless variable):

$a \quad$ convergence length of cross-sectional area.

$\bar{A} \quad$ tidally averaged cross-sectional area of flow.

$\overline{A_{0}} \quad$ tidally averaged cross-sectional area at the$$
\bar{B} \quad \text { estuary mouth. }
$$

$B_{S} \quad$ storage width.

$c \quad$ wave celerity.

$c_{0} \quad$ celerity of a frictionless wave in a prismatic channel.

$f$ friction factor accounting for the difference in friction at $\mathrm{HW}$ and $\mathrm{LW}$.

$\underline{g} \quad$ acceleration due to gravity.

$h \quad$ tidal average depth.

$\bar{h}_{\text {critical critical depth corresponding with maximum }}$ amplification.

$K \quad$ manning-Strickler friction factor.

$r_{S} \quad$ storage width ratio.

$T \quad$ tidal period.

$x, x^{*} \quad$ longitudinal coordinate.

$\gamma \quad$ estuary shape number.

$\delta \quad$ damping number.

$\varepsilon \quad$ phase lag between HW and HWS

(or LW and LWS).

$\zeta \quad$ tidal amplitude to depth ratio.

$\zeta_{\text {inf }} \quad$ asymptotic tidal amplitude to depth ratio.

$\eta, \eta^{*} \quad$ tidal amplitude.

$\eta_{0}, \eta_{0}^{*} \quad$ tidal amplitude at the seaward boundary.

$\eta_{\text {inf }}, \eta_{\text {inf }}^{*}$ asymptotic tidal amplitude.

$\phi_{z}, \phi_{U} \quad$ phase of water level and velocity.

$\lambda$ celerity number.

$\mu \quad$ velocity number.

$\mu_{0} \quad$ velocity number at the seaward boundary.

v tidal velocity amplitude.

$v_{0} \quad$ tidal velocity amplitude at the seaward boundary.

$v_{\text {inf }} \quad$ asymptotic velocity amplitude.

$\chi \quad$ friction number.

$\chi_{0} \quad$ friction number at the seaward boundary.

$\omega \quad$ tidal frequency.

$\Delta x \quad$ length step.

[75] Acknowledgments. We wish to acknowledge the contribution made by Nicolas Zimmermann who worked on the analytical solution of the tidal amplitude during his master studies at TU-Delft and who was the first to derive an asymptotic solution of the tidal amplitude. The first author is financially supported during his $\mathrm{PhD}$ program by the China Scholarship Council with the project reference of 2010638037. The Ministry of Infrastructure and the Environment of the Netherlands (Rijkswaterstaat) and the Department of Mobility and Public Works (MOW) of the
Flemish Government are gratefully acknowledged for supplying the hydrodynamic and bathymetric data of the Scheldt estuary.

\section{References}

Cai, H., H. H. G. Savenije, and M. Tofflon (2012a), A new analytical framework for assessing the effect of sea-level rise and dredging on tidal damping in estuaries, J. Geophys. Res., 117, C09023, doi:10.1029/ 2012JC008000

Cai, H., H. H. G. Savenije, Q. Yang, S. Ou, and Y. Lei (2012b), Influence of river discharge and dredging on tidal wave propagation: Modaomen estuary case, J. Hydraul. Eng., 138, 885-896, doi:10.1061/ (ASCE)HY.1943-7900.0000594.

Dronkers, J. J. (1964), Tidal Computations in River and Coastal Waters, 518 pp., Elsevier, New York.

Friedrichs, C. T. (2010), Barotropic tides in channelized estuaries, in Contemporary Issues in Estuarine Physics, edited by A. Valle-Levinson, pp. 27-61, Cambridge Univ. Press, Cambridge, U. K.

Friedrichs, C. T. and D. G. Aubrey (1994), Tidal propagation in strongly convergent channels, J. Geophys. Res., 99(C2), 3321-3336.

Horrevoets, A. C., H. H. G. Savenije, J. N. Schuurman, and S. Graas (2004), The influence of river discharge on tidal damping in alluvial estuaries, J. Hydrol., 294(4), 213-228.

Hunt, J. N. (1964), Tidal oscillations in estuaries, Geophys. J. R. Astron. Soc., 8(4), 440-445.

Ippen, A. T. (1966), Tidal dynamics in estuaries, part I: Estuaries of rectangular section, in Estuary and Coastline Hydrodynamics, edited by A. T. Ippen, pp. 493-522. McGraw Hill, New York.

Jay, D. A. (1991), Green law revisited-Tidal long-wave propagation in channels with strong topography, J. Geophys. Res., 96(C11), 20,585-20,598.

Jay, D. A. and E. P. Flinchem (1997), Interaction of fluctuating river flow with a barotropic tide: A demonstration of wavelet tidal analysis methods, J. Geophys. Res., 102(C3), 5705-5720, doi:10.1029/96JC00496.

Lanzoni, S., and G. Seminara (1998), On tide propagation in convergent estuaries, J. Geophys. Res., 103(C13), 30,793-30,812.

Lorentz, H. A. (1926), Verslag Staatscommissie Zuiderzee (in Dutch), Algemene Landsdrukkerij, Hague, Netherlands.

Prandle, D. (2003), Relationships between tidal dynamics and bathymetry in strongly convergent estuaries, J. Phys. Oceanogr., 33(12), 2738-2750.

Savenije, H. H. G. (1992a), Lagrangian solution of St. Venants equations for alluvial estuary, J. Hydraul. Eng., 118(8), 1153-1163.

Savenije, H. H. G. (1992b), Rapid assessment technique for salt intrusion in alluvial estuaries, $\mathrm{PhD}$ thesis, Int. Inst. for Infrastructure, Hydraul. and Environ., Delft, Netherlands.

Savenije, H. H. G. (1993), Determination of estuary parameters on basis of Lagrangian analysis, J. Hydraul. Eng., 119(5), 628-642.

Savenije, H. H. G. (1998), Analytical expression for tidal damping in alluvial estuaries, J. Hydraul. Eng., 124(6), 615-618.

Savenije, H. H. G. (2001), A simple analytical expression to describe tidal damping or amplification, J. Hydrol., 243(3-4), 205-215.

Savenije, H. H. G. (2005), Salinity and Tides in Alluvial Estuaries, 107 pp., Elsevier, New York.

Savenije, H. H. G. (2012), Salinity and Tides in Alluvial Estuaries, 100 pp., completely revised 2nd ed., Elsevier, New York. [Available at www.salinityandtides.com.]

Savenije, H. H. G., and E. J. M. Veling (2005), Relation between tidal damping and wave celerity in estuaries, J. Geophys. Res., 110, C04007, doi:10.1029/2004JC002278.

Savenije, H. H. G., M. Toffolon, J. Haas, and E. J. M. Veling (2008), Analytical description of tidal dynamics in convergent estuaries, J. Geophys. Res., 113, C10025, doi:10.1029/2007JC004408.

Souza, A. J., and A. E. Hill (2006), Tidal dynamics in channels: Single channels, J. Geophys. Res., 111, C09037, doi:10.1029/2006JC003469.

Toffolon, M., and H. H. G. Savenije (2011), Revisiting linearized onedimensional tidal propagation, J. Geophys. Res., 116, C07007, doi: 10.1029/2010JC006616.

Toffolon, M., G. Vignoli, and M. Tubino (2006), Relevant parameters and finite amplitude effects in estuarine hydrodynamics, J. Geophys. Res., 111, C10014, doi:10.1029/2005JC003104.

Van Rijn, L. C. (2011), Analytical and numerical analysis of tides and salinities in estuaries; part I: Tidal wave propagation in convergent estuaries, Ocean Dyn., 61, 1719-1741, doi:10.1007/s10236-011-0453-0. 